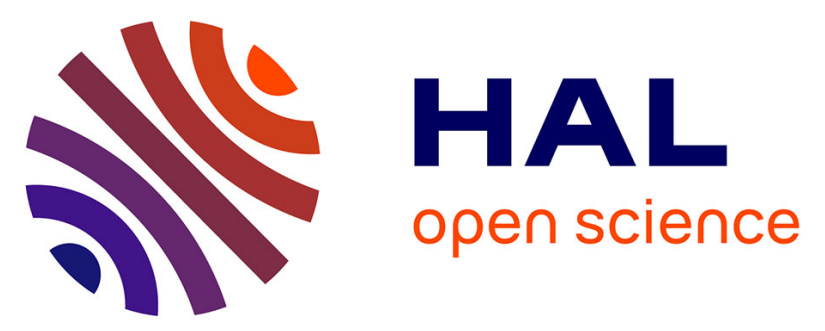

\title{
A Review on Conductive Polymers and Their Hybrids for Flexible and Wearable Thermoelectric Applications
}

Geoffrey Prunet, Florent Pawula, Guillaume Fleury, Eric Cloutet, Anthony

James Robinson, Georges Hadziioannou, Amir Pakdel

\section{To cite this version:}

Geoffrey Prunet, Florent Pawula, Guillaume Fleury, Eric Cloutet, Anthony James Robinson, et al.. A Review on Conductive Polymers and Their Hybrids for Flexible and Wearable Thermoelectric Applications. Materials Today Physics, 2021, pp.100402. 10.1016/j.mtphys.2021.100402 . hal-03188097

\section{HAL Id: hal-03188097 https://hal.science/hal-03188097}

Submitted on 1 Apr 2021

HAL is a multi-disciplinary open access archive for the deposit and dissemination of scientific research documents, whether they are published or not. The documents may come from teaching and research institutions in France or abroad, or from public or private research centers.
L'archive ouverte pluridisciplinaire HAL, est destinée au dépôt et à la diffusion de documents scientifiques de niveau recherche, publiés ou non, émanant des établissements d'enseignement et de recherche français ou étrangers, des laboratoires publics ou privés. 


\section{A Review on Conductive Polymers and Their Hybrids for Flexible and}

\section{Wearable Thermoelectric Applications}

Geoffrey Prunet ${ }^{1}$, Florent Pawula ${ }^{1}$, Guillaume Fleury ${ }^{1,{ }^{*}}$, Eric Cloutet ${ }^{1}$, Anthony James Robinson ${ }^{2}$, Georges Hadziioannou ${ }^{1}$, Amir Pakdel ${ }^{2, *}$

${ }^{1}$ Laboratoire de Chimie des Polymères Organiques (LCPO), CNRS UMR 5629 - Université de Bordeaux ENSCBP, F-33607, Pessac, France

${ }^{2}$ Department of Mechanical, Manufacturing \& Biomedical Engineering, Trinity College Dublin, The University of Dublin, D02PN40 Dublin, Ireland

*Correspondence: gfleury@enscbp.fr (GF); pakdela@tcd.ie (AP)

\section{Graphical Abstract:}

\section{Abstract:}

There is a growing demand for flexible and wearable next-generation electronic devices that must be capable of bending and stretching under mechanical deformation. In this regard, energy harvesting technologies have immensely invested in organic and polymeric semiconducting materials due to their large-area synthesis, low cost, low toxicity, high flexibility, and tunable electronic properties. For example, electrically conductive $\pi-$ conjugated polymers have been investigated in various thermoelectric technologies for producing stretchable, wearable, and light-weight thermoelectric devices that can harvest energy from a temperature gradient and produce electricity with no pollution or moving parts. In this review we initially provide a general overview of the thermoelectric principles and conductive polymer characteristics, followed by the recent progress in their application in flexible and wearable thermoelectric devices. We also evaluate new advances in manufacturing hybrids of $\pi$-conjugated polymers with other polymers, inorganic materials, 
or carbon nanostructures, and their applications in body energy harvesting and smart cooling.

\section{Highlights}

- A review assessing state of the art flexible and wearable heat-to-electricity energy convertors.

- Categories and characteristics of thermoelectric conductive polymers and their nanohybrids.

- A physics, chemistry and technology framework for thermoelectric body heat harvesting research.

- Outlook on the organic/polymer field challenges to upscale thermoelectric energy convertors.

\section{Keywords:}

Conductive polymers, Organic thermoelectric materials, Hybrid thermoelectric materials, Flexible thermoelectric generators, Wearable thermoelectric devices, Body energy harvesting. 


\section{Introduction}

Since the control of fire by early humans, we have sought to diversify our means of producing thermal energy. Initially, the main source of thermal energy was biomass combustion (such as wood and animal waste). It then evolved throughout history with the use of fossil fuels. Eventually the thermal energy sources were broadened through scientific discoveries and technological progress, and today heat can be harvested from a number of sources such as: mechanical energy via friction and shocks [1], nuclear energy via fission [2], radiant energy via solar collectors [3], and even geothermal energy [4]. Nevertheless, most of the produced heat is untapped and released directly into the atmosphere [5]. In fact, around $2 / 3$ of all energy generated is not harnessed for any productive use [6]. This is exemplified by the fact that internal combustion engines are only about $20-40 \%$ efficient, and even electric engines waste $10-15 \%$ of their energy $[7,8]$.

A portion of unused thermal energy can be harvested for increasing the energy production of the systems. Among the various potential renewable energy systems, thermoelectricity is one solution capable of transforming waste heat energy directly into electricity, improving the overall thermal efficiency of the system. However, considering the relatively low power yield of the present thermoelectric devices, this form of sustainable energy recovery has not yet seen large penetration in the context of the current global energy challenge and related environmental crisis [9]. In fact, considering the viable power yields, application of thermoelectric generators (TEGs) can be more promising in low-power personal devices. Particularly, small and flexible TEGs can provide the $\mu \mathrm{W}-\mathrm{mW}$ power requirement of smart clothes, health monitoring sensors, and biosensors. For applications that require larger power supplies such as cellphones, fitness trackers, and smart watches, TEGs can be coupled with microelectronic booster circuits or storage devices (e.g. capacitors or batteries) that nowadays are as thin as a paper sheet. Nonetheless, the wearability of TEGs is still in its infancy, as a diversity of challenges (environmental stability, reliability to mechanical fatigue, proper integration in devices, optimized power conversion efficiency) still needs to be addressed in order to achieve practical and sustainable devices. Answering these challenges undoubtedly require deeper scientific knowledge and additional experimental studies in molecular and macromolecular chemistry, solid-state/soft-matter 
physics, materials science, manufacturing engineering, as well as physiology, i.e. from chemical formulation to device design and integration.

In this regard, researchers have been constantly trying to achieve a significant improvement in the efficiency of thermoelectric devices. Currently, TEGs are mainly made from inorganic semiconducting materials due to their superior thermoelectric properties [10]. However, some of these inorganic materials consist of elements with low abundance in earth's crust [11]. Moreover, their relatively heavy weight is a limiting factor for embedded applications and their low processability, high toxicity and high cost (e.g. around $\$ 800 / \mathrm{kg}$ for bismuth telluride) are problematic for the future device uses [12]. It was in this scenario that the discovery of efficient charge transport in doped conjugated polymers ( $p$ - and n-type) [1315] attracted the attention of the thermoelectric scientific community in its search for innovative and competing materials for TEGs $[16,17]$. Indeed, the conducting polymers' strengths lie in their intrinsic low thermal conductivity, high processability (printable techniques applicable), and low cost [18-21]. However, the low electrical conductivity, in particular for n-type polymers, as compared to inorganic thermoelectric materials, is the main reason behind their inferior efficiency. Thus, their application as thermoelectric materials is still limited to niche applications that involve low-powered devices. Nonetheless, strategies to reach relatively high electrical conductivity based on structural modification and band gap tuning have been introduced and are discussed in the sections that follow.

Electrically conductive $\pi$-conjugated polymers are carbon-based materials consisting of alternated simple and double (or triple) bonds that allow charge transport. Important aspects, such as the macromolecular architecture, doping level, and crystallinity of these material strongly influence their electronic states density. For example, extending the degree of polymerization of $\pi$-conjugated polymer chains causes a broadening of the $\pi$ orbitals with a pronounced overlapping, which leads to a modification of the density of states from isolated states to a band-like structure. Hence, $\pi$-conjugated polymers can electronically range from semiconductors, semimetals to metals. Also, the electrical properties of such polymers are highly versatile as they are heavily influenced by the processing methodology. 
In the following sections, we will review the thermoelectric principles, the properties that affect the thermoelectric behavior of a material, the conductive polymers characteristics and their application in flexible and wearable thermoelectric devices. Finally, we will highlight recent advances in manufacturing hybrids of conducting polymers with other polymers, inorganic materials, and carbon nanostructures.

\subsection{Thermoelectric principles}

Thermoelectricity combines two phenomena in a material; heat transfer ("thermo") and charge transfer ("electricity"). Thermoelectric systems can either convert directly heat into electricity or convert an electrical power into a cooling source. The performance of thermoelectric materials is characterized by the Figure of Merit $Z T=S^{2} \sigma / K$, a dimensionless parameter first introduced by Edmund Altenkirch in 1911 [22]. The Figure of Merit at a given temperature $T$, contains the Seebeck coefficient $S$, the electrical conductivity $\sigma$, and the thermal conductivity $\mathrm{K}$. The product in the numerator, $\mathrm{S}^{2} \sigma$, is known as the Power Factor (PF) and is sometimes used to compare the performance of thermoelectric materials, especially when it is difficult to measure the thermal conductivity (for instance in thin films).

\section{Seebeck coefficient}

Applying a temperature difference $(\Delta T)$ across a conductive or semiconducting material generates a voltage potential $(\Delta \mathrm{V})$. The proportionality between $\Delta \mathrm{V}$ and $\Delta \mathrm{T}$ is given by an intrinsic material property known as the Seebeck coefficient $S=-\Delta V / \Delta T$. By convention, for semiconductors, the Seebeck coefficient is negative for $n$-type and positive for $p$-type materials. Thus, the Seebeck effect is of paramount importance in the semiconductor field, as it allows for the determination of the type of dominating charge carriers in a material as well as the relative positioning of the Fermi level with respect to the transport levels [23].

\section{Electrical conductivity}

Electrical conductivity $(\sigma)$ is also a key parameter in the thermoelectric field. It is a material-inherent property for conducting current, and is linked to charge carrier transport phenomena [24]. In semiconductors, the electrical conductivity is calculated by the following relation: $\sigma=q\left(\mu_{n} n+\mu_{p} p\right)$, where $\mu_{n}\left(\mu_{p}\right)$ is the mobility of electrons (holes), $n(p)$ is the charge carrier concentration of electrons (holes), and $q$ is the elementary charge. 
Thermal conductivity

The last important parameter that is involved in thermoelectricity is the thermal conductivity [24]. The Thermal conductivity ( $\mathrm{k}$ ) is the ability to transfer heat without matter displacement that can be defined as a ratio of heat flux density to temperature gradient based on Fourier's law. The thermal energy transfer through conduction is a random process depending on diffusivity and particles collision in a material. Considering metals and heavily doped inorganic semiconductors, there are two parallel contributions to $\mathrm{k}$ : the structural $\mathrm{ks}$ related to lattice/backbone collective vibrations which can be described by quasi-particles like phonons, locons, propagons, and the electronic contribution Ke related to the material's charge carriers that also influence the propagation of phonons and thus contribute to the heat transport capacity $[25,26]$.

Improving the thermoelectric performance is fundamentally challenging because $S$ and $\sigma$ are antagonistically interdependent, and also coupled to $\mathrm{K}$. The ZT relationship suggests that to improve the performance one might aim to enhance the electrical conductivity, for example, by increasing the carrier concentration, $n(\sigma=n q \mu)$. However, increasing the carrier concentration generally leads to a reduction in the material's Seebeck coefficient and an increase in the thermal conductivity. Therefore, ZT improvement is difficult to achieve and usually limited to the optimization of charge carrier levels in doped semiconductors or semimetals in order to balance the thermal conductivity and the electronic transport properties [27-31]. An ideal thermoelectric material is often described as a "phonon-glass electron-crystal", denoting a low $\mathrm{k}$, high $\mathrm{S}$ and high $\sigma$ [31]. Regarding inorganic compounds, the best thermoelectric materials are traditionally bismuth telluride alloys ( $\mathrm{Bi}-\mathrm{Te}$ ) at room temperature $(\mathrm{ZT}=1.86$ at $320 \mathrm{~K})$ and lead telluride $(\mathrm{Pb}-\mathrm{Te})$ at high temperature $(\mathrm{ZT}=2.2$ at $915 \mathrm{~K}$ ) [32]. Regarding organic compounds, the best thermoelectric performance reported so far at room temperature is $\mathrm{ZT}=0.75$ with a power factor of $754 \mu \mathrm{W} \cdot \mathrm{m}^{-1} \cdot \mathrm{K}^{-2}$ in poly $(3,4-$ ethylenedioxythiophene):poly(styrene sulfonate) (PEDOT:PSS) thin films with 1-ethyl-3methylimidazolium dicyanamide (EMIM-DCA) ionic liquids at the film surface [33]. So far, the inorganic thermoelectric materials have exhibited a much higher ZT, but their organic counterparts (and in particular polymers) have other intrinsic advantages like flexibility, low cost, scalability, and light weight, which are essential for flexible, portable, and wearable applications. 


\section{Afterword on transport properties}

In a non-degenerate semiconductor, the Seebeck coefficient is inversely proportional to the
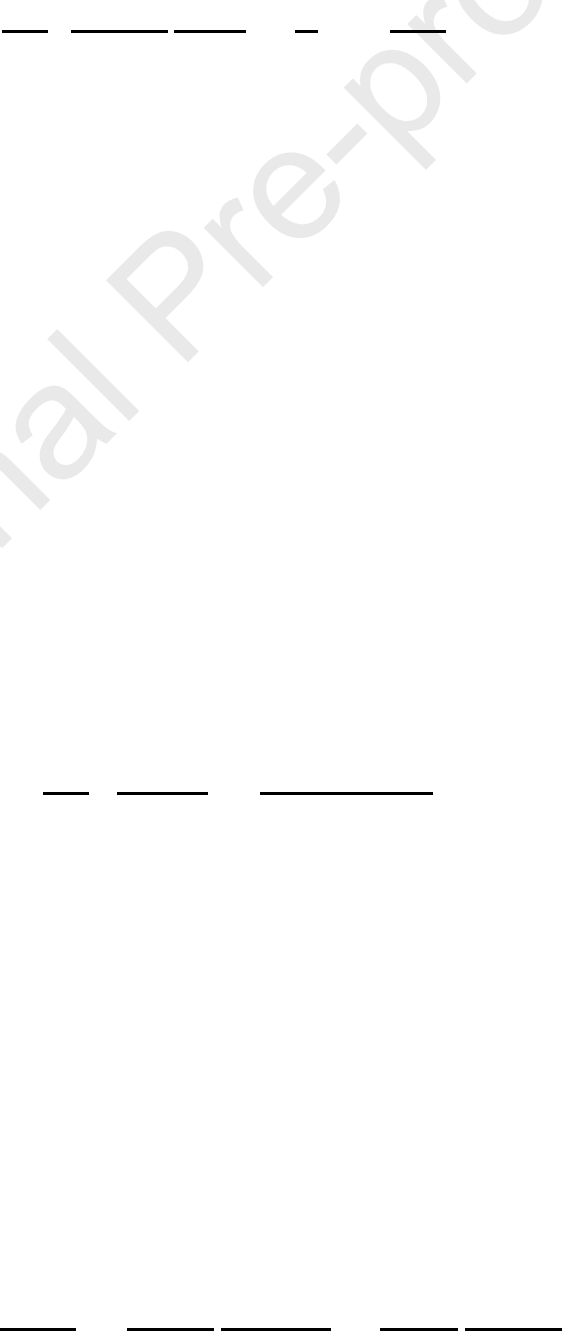
and is related to the charge carrier density $(n)$ through $n=\int N(\varepsilon) f(\varepsilon) d \varepsilon$, in which $f(\varepsilon)$ is the Fermi-Dirac distribution function [36].

In summary, the Seebeck coefficient and electrical conductivity of conjugated polymers strongly depend on the electronic band structure, i.e. the charge carrier density and mobility. Additionally to the macromolecular design of conductive polymers which virtually affords any combination of chemistry and sequencing, multiple approaches have been reported to further tune their band gap energy, DOS, charge carrier density and mobility, e.g. structural engineering, synthetic formulation or tuning of the doping level. These approaches illustrated by recent reports to control the Seebeck coefficient and electrical conductivity of conjugated polymers for thermoelectric applications are discussed for various promising materials in Section 2.

\section{Conductive polymer materials for flexible thermoelectric applications}

\subsection{Motivation}

Over recent years, interest in conductive polymers for thermoelectric applications has escalated thanks to their unique combination of properties [37]. Semiconducting polymers can exhibit a high electrical conductivity of $\sim 1000{\mathrm{~S} . \mathrm{cm}^{-1}}^{-138]}$ and a relatively low thermal conductivity of $\sim 0.1-1 \mathrm{~W} \cdot \mathrm{m}^{-1} \cdot \mathrm{K}^{-1}$ as compared to that of conventional inorganic semiconductors ( 1-100 W.m $\left.{ }^{-1} \cdot K^{-1}\right)$ [39-43]. Furthermore, polymers have the major advantages of being printable, flexible, moldable and easily scalable which are non-negligible advantages [16, 43]. However, as the Seebeck coefficient of these polymers is not intrinsically high, they have demonstrated lower thermoelectric efficiencies than their inorganic counterparts. In the next sections, different strategies to enhance the thermoelectric properties of $\pi$-conjugated polymers ( $p$ - and $n$-type) will be discussed.

\subsection{P-type conductive polymers}

Conductive polymers were not initially anticipated as potential thermoelectric materials. The first widely studied electrically-conductive polymer is trans-polyacetylene characterized by the intrinsic planarity of its backbone [44-47]. When doped by arsenic pentafluoride (AsF5), relatively high conductivities of $560 \mathrm{~S} . \mathrm{cm}^{-1}$ and carrier mobilities of $\sim 1 \mathrm{~cm}^{2} \cdot \mathrm{V}^{-1} \cdot \mathrm{s}^{-1}$ 
were measured, but low Seebeck values $\sim 10 \mu \mathrm{V} . \mathrm{K}^{-1}$ were measured for high carrier concentrations [48]. Nevertheless, 30 years later, the potential of polymers as thermoelectric materials was demonstrated by Hiroshige et al. by doping poly(p-phenylene vinylene) (PPV) with iodine (ZT = 0.1) [49].

Today, the representative p-type thermoelectric polymers include PEDOT, polythiophene derivatives (more particularly poly(3-hexylthiophene, $\mathrm{P3HT}$ ), polyaniline (PANI), polypyrrole (PPy), polyphenylenevinylene (PPV), polycarbazole (like poly[N- 9'-heptadecanyl-2,7carbazole-alt-5,5-(4,7-di-2-thienyl-2',1',3'-benzothiadiazole], PCDTBT) and poly(benzodithiophene) (like Poly[[4,8-bis[(2-ethylhexyl)oxy]benzo[1,2-b:4,5-b']dithiophene2,6-diyl][3-fluoro-2-[(2-ethylhexyl) carbonyl] thieno[3,4-b]thiophenediyl]], PTB7) (Figure 1).

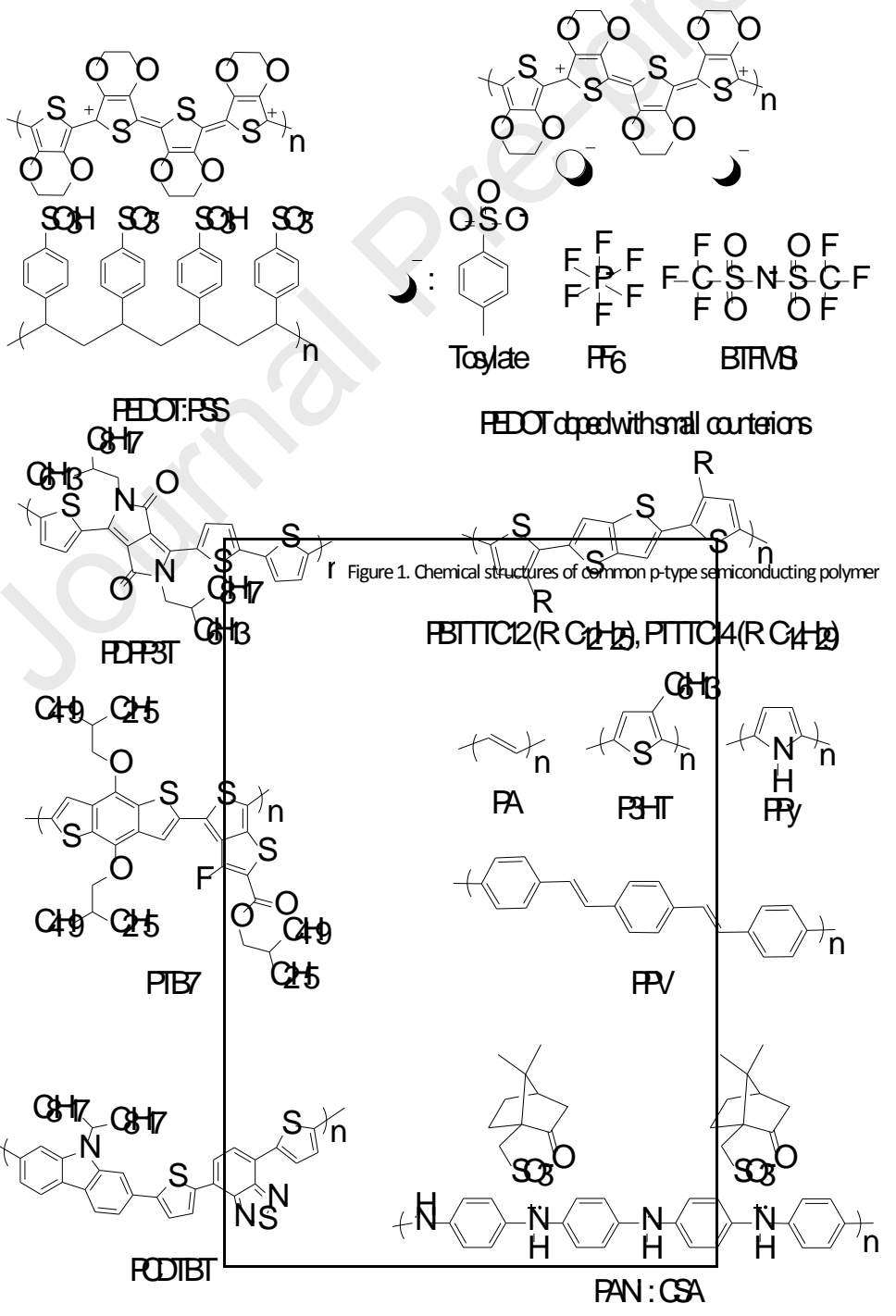




\subsubsection{PEDOT-based p-type thermoelectric materials}

The PEDOT family is the most studied and developed p-type thermoelectric material. Among those family members, PEDOT:poly(styrenesulfonate) (PEDOT:PSS) and PEDOT:ptoluenesulfonate (PEDOT:TOS) have received the greatest attention so far [50-52]. PEDOT:PSS is water dispersible and the suspensions are commercially available. As-prepared PEDOT:PSS films have low thermoelectric conversion efficiency with electrical conductivities lower than 1 S.cm ${ }^{-1}$ and Seebeck coefficients of $~ 15 \mu \mathrm{V} . \mathrm{K}^{-1}$ [53-61]. These low properties are related to an excess of non-conductive PSS chains, necessary to stabilize PEDOT in water [54]. The thermoelectric properties, particularly the electrical conductivity, can be enhanced by removing the excess PSS (i.e. dedoping) by various methods developed recently [61-70]. Furthermore, the Seebeck coefficient can be improved with the addition of structural agents leading to an enhanced crystallinity [53]. Similar to PEDOT:PSS, in-situ polymerized PEDOT:Tos has also received great attention thanks to its good electrical performance, easy fabrication, and property tunability improved by chemical dedoping and structural engineering [71-75].

\subsubsection{Structural engineering and morphology tuning}

In semi-crystalline conductive polymers, the electronic band structure can be tuned through macromolecular engineering and refined further via adequate structuration into highly ordered crystalline regions. Therefore, structural engineering is as also called "secondary doping", since it allows an improvement of the electronic properties [76]. In principle, a higher degree of crystallinity leads to an improved electrical conductivity in a material as the charge transport is facilitated $[77,78]$. When a conductive polymer film is treated with a solvent (in either liquid or vapor form), its morphology is modified without altering the effective doping level. For instance, the PEDOT:PSS thermoelectric performance can be enhanced by adding poly(ethylene oxide) (PEO), ethylene glycol (EG), or dimethyl sulfoxide (DMSO), because the resulting morphological modifications improve the electrical conductivity with a concomitant minor reduction of the Seebeck coefficient $[63,64,79-81]$.

Although PEDOT:PSS has the potential for very high electrical conductivity, it suffers from a small ionization fraction induced by the PSS dopant in its pristine form. Consequently, a large excess of PSS is required to create a high carrier concentration. Such high carrier 
concentration samples yield low values of S, highlighting the need to control the PSS amount in PEDOT:PSS dispersions. Moreover, the presence of many non-ionized dopants can considerably decrease the carrier mobility and consequently the Seebeck coefficient [82]. Accordingly, Kim et al. demonstrated how high boiling point solvents, such as ethylene glycol (EG) and dimethyl sulfoxide (DMSO), favorably influence the PEDOT:PSS thermoelectric properties [83]. PEDOT:PSS films were treated in EG and DMSO baths to wash out the excess PSS, and led to the improvement of the power factor from 50 to $469 \mu \mathrm{W} \cdot \mathrm{m}^{-1} \cdot \mathrm{K}^{-2}$. It is because the superfluous PSS chains in PEDOT:PSS films result in an extended phase separation, which is detrimental to charge transport taking place by tunneling between conductive nanoscale PEDOT-rich islands surrounded by PSS-rich shells. By removing the excess PSS, the tunnelling distance decreases and allows an improved charge carrier mobility, which in turn induces an increase of the Seebeck coefficient and consequentially the power factor. Later, Palumbiny et al. [84] showed that the related increase of crystallinity was induced by a reduced crystallization kinetics due to the plasticizers effect of high boiling point solvents. Hence, an improved rearrangement of the polymer chains was possible in the film configuration, leading to an increase in the degree of crystallinity which facilitated the charge carrier transport (Figure 2) [58, 68, 84]. 
Figure 2. Plasticizing agents effect on the PEDOT:PSS structure. When EG is added to PEDOT:PSS material (b), the crystallization process is slowed down resulting in a better stacking of the polymer chains, compared to the pure PEDOT:PSS material (a). When the thin films are further treated in an EG bath, the system recrystallizes and shifts from a face-one to an edge-on configuration (c) [84].

Then, Bubnova et al. proposed that PEDOT derivatives with a tailored crystallinity could show semimetallic behavior, i.e. possessing a relatively high electrical conductivity along with a relatively high Seebeck coefficient [85]. Their PEDOT:Tos films made by vapor-phase polymerization exhibited a power factor as high as $454 \mu \mathrm{W} \cdot \mathrm{m}^{-1} \cdot \mathrm{K}^{-2}$. In highly crystalline PEDOT systems, the PEDOT units $\pi$-orbitals overlap and recombine, inducing the formation of a bipolaron network, increasing the density of states toward the Fermi level $(\varepsilon F)$, and enhancing the electrical conductivity and the Seebeck coefficient. Other recent studies also support the benefit of increased crystallinity on the PEDOT derivatives thermoelectric properties in thin film [85-88]. Petsagkourakis et al. correlated the charge carrier mobility in PEDOT:Tos thin films to their crystallinity and investigated their influence on the thermoelectric properties [77]. To increase the films crystallinity, the polymer solution was 
mixed with two additives: an organic base (pyridine) and a high boiling point solvent (DMSO). X-ray photoelectron spectroscopy (XPS) investigations demonstrated how such treatment did not affect the oxidation level of the polymers; therefore, attributing the enhanced electrical conductivity to an improved charge carrier mobility. Similarly, a Seebeck coefficient enhancement with charge carrier mobility was observed and a proportional relationship between these factors was extracted as: $s \propto \mu^{0.2}$.

\subsubsection{Tuning of the doping level}

One of the main concerns about thermoelectric development is the modest electrical conductivity of intrinsically conducting polymer materials, for instance pristine PEDOT:PSS has an electrical conductivity of $0.1-0.5{\mathrm{~S} . \mathrm{cm}^{-1}}^{-189]}$. However, it has been shown that the thermoelectric properties of PEDOT films can be increased by removing the unionized portion of the counter-anion molecules, which reduces the charge carrier mobility [90]. This process is called "dedoping", although it does not change the effective doping level. This is an additional key to tune the electronic properties of polymeric thermoelectric materials, compared to inorganic materials. As a reference study, Bubnova et al. optimized the PEDOT:Tos thermoelectric properties by exposing their films to reductive vapors, such as tetrakis(dimethylamino) ethylene-TDAE [52]. By dedoping the films, the electrical conductivity was decreased as opposed to the Seebeck coefficient (Figure $3(\mathrm{a})$ ). The resulting power factor was improved from 38 to $324 \mu \mathrm{W} \cdot \mathrm{m}^{-1} \cdot \mathrm{K}^{-2}$, and a ZT of 0.25 was obtained at room temperature, based on the calculated thermal conductivity.

In a sample, the dedoping degree is strongly influenced by the reducing agent electrochemical potential and chemical nature. For instance, Massonnet et al. [91] showed that by dipping PEDOT:PSS films in TDAE or hydrazine, Seebeck coefficients of 43 and 93 $\mu \mathrm{V} . \mathrm{K}^{-1}$ were obtained, respectively. This difference is related to the chemical nature of these reducing agents. TDAE, a non-polar organic reducing agent, shows limited interactions with the hydrophilic PEDOT:PSS layer, as compared to the more polar hydrazine. Alternatively, Khan et al. [92] investigated the influence of the $\mathrm{pH}$ of treating solution, i.e. hydrochloric acid and sodium hydroxide, on PEDOT thermoelectric properties (Figure 3(b)). Treating thin film samples with acidic or basic solutions did modify the electronic transport with the highest power factor of $26 \mu \mathrm{W} \cdot \mathrm{K}^{-2} \cdot \mathrm{m}^{-1}$ for $\mathrm{pH}>7$. The authors explained that treating the material with a basic $\mathrm{pH}$ solution affects the oxidation levels leading to modification from 
bipolaronic states to polaronic states, as confirmed by UV-visible and IRAS spectra analysis. Later, Fan et al. [93] used sulfuric acid with sodium hydroxide (a reducing agent) on pristine PEDOT:PSS films. The sulfuric acid oxidized and modified the film morphology, increasing the electrical conductivity. The power factor of the PEDOT:PSS film was enhanced by 5 orders of magnitude, from $4.5 \times 10^{-3} \mu \mathrm{W} \cdot \mathrm{m}^{-1} \cdot \mathrm{K}^{-2}$ to $334 \mu \mathrm{W} \cdot \mathrm{m}^{-1} \cdot \mathrm{K}^{-2}$.

Figure 3. (a) Electrical conductivity $\sigma$, Seebeck coefficient $S$, and the power factor $\sigma S^{2}$, versus the oxidation levels of the PEDOT:Tos films. [52] (b) UV-Vis spectra of acid and base treated PEDOT:Tos with respect to the treating solution $\mathrm{pH}[92]$.

All the aforementioned works were based on conventional chemical oxidation-reduction (redox) reactions. An alternative approach to optimize the doping level in conductive polymers is based on electrochemical methods. A typical electrochemical setup is composed of three electrodes: a working, a reference and a counter electrode. This technique consists of depositing a polymer film by oxidation (anodic polymerization) on electrically conductive surfaces, e.g. gold, platinum, $\ln _{2} \mathrm{O}_{3}$ (ITO), etc. [94]. Electrochemical polymerization is a very attractive method because polymerization, doping and processing take place simultaneously. Moreover, a large choice of counter-ions is available, whose only requirement is solubility in the electrolytic solution. The main influencing factors to change the transport properties and film morphology are temperature, current density and current frequency, electrolyte nature and counter-ions for PEDOT [95]. Park et al. [96] synthesized PEDOT:TOs by this process and obtained a record power factor of $1270 \mu \mathrm{W} \cdot \mathrm{m}^{-1} \cdot \mathrm{K}^{-2}$. Another demonstration by Bubnova et al. [97] was accomplished with an organic electrochemical transistor (OECT) architecture used to further increase the oxidation level (i.e. not only on a 
sample surface, but also throughout the bulk) leading to a Seebeck coefficient of $\sim 400 \mu \mathrm{V} . \mathrm{K}^{-}$ ${ }^{1}$ in PEDOT:PSS samples.

\subsubsection{Non PEDOT-based p-type thermoelectric materials}

Historically, the first conductive polymer was trans-polyacetylene [44, 45, 48], a "nonPEDOT-based" p-type polymer. Although PEDOT:PSS and derivatives are widely represented in the thermoelectric field, considerable research on other p-type polymers has been performed as well. These studies aimed at improving the thermoelectric properties of p-type polymers through an optimization of their morphology and doping level.

\subsubsection{Polyacetylene (PA)}

PA is an archetypal conjugated polymer that is made of a long chain of carbon atoms with alternating single and double bonds. When polyacetylene is doped with halogens such as chlorine $(\mathrm{Cl})$, bromine $(\mathrm{Br})$ or iodine $(\mathrm{I})$ vapors or with arsenic pentafluoride $\left(\mathrm{AsF}_{5}\right)$, an increase in the electrical conductivity to $2 \times 10^{4} \mathrm{~S} . \mathrm{cm}^{-1}$ can be achieved [44, 98-100]. There are also reports demonstrating that stretching can enhance its conductivity because of the increased crystallinity due to the shear alignment of the polymer backbone. For instance, the electrical conductivity of an iodine-doped PA film was doubled from $3 \times 10^{3}$ to $6 \times 10^{3}{\mathrm{~S} . \mathrm{cm}^{-1}}^{-1}$ upon stretching [101]. Zuzok et al. reported the PA thermoelectric properties doped with metal halides such as $\mathrm{FeCl}_{3}, \mathrm{ZrCl}_{4}$ and $\mathrm{NbCl}_{5}[101,102]$. They demonstrated that the huge intrinsic conductivity of metallic regions in PA is related to an unusually small electronphonon interaction for carriers involved in charge transport. Despite being the first polymer showing a good electrical conductivity of $44 \mathrm{~S} . \mathrm{cm}^{-1}$ and the highest power factor of $9.9 \times 10^{-4}$ $\mu \mathrm{W} \cdot \mathrm{m}^{-1} \cdot \mathrm{K}^{-2}$ after doping [103], PA has not been very popular in organic thermoelectric devices, because of its poor solubility and high oxidation tendency in air. Nevertheless, the promising thermoelectric properties of PA acted as the driving force for research on other more stable p-type materials in air conditions.

\subsubsection{Polyaniline (PANI)}

PANI's popularity as a conducting polymer is due to its facile synthesis and interesting properties such as chemical stability, flexibility and solution processability. Another inherent property of PANI is its ability to be p-or n-doped, i.e. the main charge carrier type in PANI can be controlled through the $\mathrm{pH}$ tuning of dopants or by the grafting of organic/inorganic 
moieties on the polymer chains [104-106]. Furthermore, the synthesis of PANI can be performed chemically or electrochemically, resulting in materials with different morphologies and electrical conductivities [107]. This is because the electrical conductivity of PANI depends on its oxidation state; and as PANI has three distinct oxidation states and acid/base doping response, its electrical conductivity can be tuned from $10^{-7}$ to $3 \times 10^{2}{\mathrm{~S} . \mathrm{cm}^{-1}}^{-1}$ $[108,109]$.

The dependence of $\mathrm{HCl}$-doping concentration on the thermoelectric properties of PANI was studied by Li et al. and a maximum $Z T$ value of $2.7 \times 10^{-4}$ at $423 \mathrm{~K}$ was achieved [80]. Nath et al. used camphor sulphonic acid (CSA) to dope nanocrystalline PANI and obtained a high $Z T$ value of 0.77 and 2.17 at $45 \mathrm{~K}$ and $17 \mathrm{~K}$, respectively [110]. An alternative strategy to increase the thermoelectric properties of PANI is the cross-linking of polymer chains. It has been shown that due to a better crystallinity and charge transport in the aforementioned case, the mobility and conductivity increased by almost $25 \%$ as compared to the linear polymer [111].

\subsubsection{Polypyrrole (PPy)}

PPy is another popular conducting polymer for thermoelectric purposes due to its reasonable electrical conductivity and mechanical stability in the form of flexible films. There are various ways to fabricate flexible conducting PPy films, e.g. electrochemically or oxidatively with iron(III) trichloride $\left(\mathrm{FeCl}_{3}\right)$ [112-114]. One of the advantages of the electrochemical route is the possibility to dope the polymer chains during the process, for example by $\left(\mathrm{Fe}(\mathrm{CN})_{6}\right)^{3-}$ [115]. Another attractive method is the freezing interfacial polymerization, which allows to obtain high electrical conductivity of $2 \times 10^{3} \mathrm{~S}_{\mathrm{cm}} \mathrm{cm}^{-1}$ due to an increased ordering of the polymeric structure [113]. However, the main drawback of this type of polymers is their low Seebeck coefficient at room temperature [116]. The highest reported value of power factor for PPy is $3.9 \mu \mathrm{W} \cdot \mathrm{m}^{-1} \cdot \mathrm{K}^{-2}[117]$.

\subsubsection{Poly(3-hexylthiophene) (P3HT)}

Regioregular P3HT is an alkylated derivative of polythiophene, and one of the widely investigated $\pi$-conjugated electrically conductive polymers. It shows a good solubility in a variety of organic solvents enhancing its film processing properties, and it is commercially available [118]. As P3HT was used with success in all-organic solar cells and all-organic field 
effect transistors, research groups investigated this particular polymer as thermoelectric material. Crispin et al. evaluated P3HT films thermoelectric properties doped with nitrosonium hexafluorophosphate $\left(\mathrm{NOPF}_{6}\right)$ [47]. The maximum performance was observed in sample with 20 to $31 \%$ doping levels, giving a power factor of $14 \mu \mathrm{W} \cdot \mathrm{m}^{-1} \cdot \mathrm{K}^{-2}$. It was demonstrated that the bulky $\mathrm{PF}_{6}{ }^{-}$anions inhibit the formation of crystallites at low doping levels, but with increasing the doping levels the structure order increases in line with the electrical conductivity, whilst the Seebeck coefficient diminishes. Similarly, Zhang et al. doped P3HT with ferric salt of TFSI and measured a power factor of $20 \mu \mathrm{W} \cdot \mathrm{m}^{-1} \cdot \mathrm{K}^{-2}[119]$.

Another recent approach in doping P3HT films is by using organic molecular dopants, such as the electron acceptor 2,3,5,6-tetrafluoro-7,7,8,8-tetracyanoquinodimethane ( $F_{4} T C N Q$ ) [120-122]. Molecular dopants induce ground-state charge carrier transfer in the semiconductor polymer host, creating polarons or bipolarons [122-124]. These dopant anions remain in the film and often result in a drastically reduced solubility. Therefore, aggregation is a major issue in polymer:dopant liquid mixtures. To avoid such phenomena, solutions shall be diluted and kept at high temperature [125]. With a conventional solution processing, an electrical conductivity of $4 \times 10^{-4} \mathrm{~S} . \mathrm{cm}^{-1}$ and a power factor of $6 \times 10^{-3} \mu \mathrm{W} . \mathrm{m}^{-1} \cdot \mathrm{K}^{-}$ ${ }^{2}$ were demonstrated [126]. Another problem with such bulk doping methods is that large amounts of $\mathrm{F}_{4}$ TCNQ negatively affect the morphology of the P3HT film and lead to $\mathrm{F}_{4} \mathrm{TCNQ}$ aggregation, which prevents achieving higher conductivity levels [127].

In a study comparing the electronic properties and surface morphologies of P3HT:F 4 TCNQ films doped directly in solution or by sequential doping processes (Figure 4) [121], Jacobs et al. observed that the sequential doping technique not only facilitates their processing, but also increases considerably the electrical conductivity at equivalent doping ratio. A 3 to 15fold higher conductivity was assigned to the optimization of the morphology with numerous tie molecules in-between crystallites as shown in Figure 4 . In a film processed by the sequential doping technique, a conductivity of $5.5 \mathrm{~S} . \mathrm{cm}^{-1}$ and a PF of $8 \mu \mathrm{W} \cdot \mathrm{m}^{-1} \cdot \mathrm{K}^{-2}$ were measured [127-129]. This procedure was further adapted to obtain thick films (>1 $1 \mu \mathrm{m})$ with almost the same properties as thin films, i.e. $\sigma$ of $3 \mathrm{~S} \cdot \mathrm{cm}^{-1}$ and PF of $5 \mu \mathrm{W} \cdot \mathrm{m}^{-1} \cdot \mathrm{K}^{-2}$. Thick films were obtained by stacking of alternated polymer and dopant layers [129]. 
Alternatively, Hynynen et al. used the sequential doping method, by exposing P3HT films to F 4TCNQ vapor, and demonstrated that the crystalline structure of the films could be controlled by the doping level [123]. They additionally showed that the molecular weight of P3HT did not strongly affect the electrical conductivity and reported a power factor of 2.7 $\mu \mathrm{W} \cdot \mathrm{m}^{-1} \cdot \mathrm{K}^{-2}$ at room temperature with a maximum Seebeck coefficient of $63 \mu \mathrm{V} \cdot \mathrm{K}^{-1}$ and electrical conductivity of 12.7 S.cm ${ }^{-1}$ [125].

Figure 4. Schematic of (a) mixed solution doping and (b) sequential doping methods to dope P3HT with F4TCNQ. The resulting morphological structures are illustrated for both methods [121]. 


\subsubsection{Donor-acceptor p-type polymer}

The co-polymerization of donor and acceptor units allows a precise tuning of the electronic properties in semiconducting polymers. There have been some studies to evaluate the thermoelectric properties of these donor-acceptor copolymers. However, doping methods and processability have not yet been fully explored in this class of polymers.

\subsection{Poly(2,5-bis(3-alkylthiophen-2-yl)thieno[3,2-b]thiophene) (PBTTT)}

PBTTT is a semiconducting $\pi$-conjugated polymer used as a hole transporting material. When it has a high degree of crystallinity, it can show a high charge carrier mobility. One of the first attempt to improve the thermoelectric properties of PBTTT was performed by doping as-cast and annealed PBTTT films using a chemical oxidant NOPF 6 :acetronitrile solution [130]. Based on spectroscopic measurements, it was found that the doping efficiency was similar for both types of films. However, the thermoelectric properties of the annealed PBTTT films ( $\mathrm{S}=13.5 \mu \mathrm{V} . \mathrm{K}^{-1}$ and $\left.\sigma=55 \mathrm{~S} . \mathrm{cm}^{-1}\right)$ were higher than those of the ascast films $\left(S=11 \mu \mathrm{V} . \mathrm{K}^{-1}\right.$ and $\left.\sigma=33 \mathrm{~S} . \mathrm{cm}^{-1}\right)$. The power factor of annealed films was three times higher, which was attributed to the increase of structural ordering [130]. Later, acid dopants like (tridecafluoro-1,1,2,2-tetrahydrooctyl)trichlorosilane (FTS) and 4-ethylbenzenesulfonic acid (EBSA) were used on PBTTT films [131], and yielded to an improvement of the conductivity, from $5 \mathrm{~S} . \mathrm{cm}^{-1}$ to $1 \times 10^{3} \mathrm{~S} . \mathrm{cm}^{-1}$ and $1.3 \times 10^{3} \mathrm{~S} . \mathrm{cm}^{-1}$, respectively. However, as the two aforementioned doping agents led to different Seebeck coefficients ( $33 \mu \mathrm{V} . \mathrm{K}^{-1}$ for FTS and $25 \mu \mathrm{V} . \mathrm{K}^{-1}$ for EBSA), the resulting power factors were significantly different; 110 $\mu \mathrm{W} \cdot \mathrm{m}^{-1} \cdot \mathrm{K}^{-2}$ for FTS and $25 \mu \mathrm{W} \cdot \mathrm{m}^{-1} \cdot \mathrm{K}^{-2}$ for EBSA. To investigate the influence of the dopant nature on the crystallographic structure, grazing-incidence wide-angle $\mathrm{X}$-ray scattering (GIWAXS) analysis was performed and it was demonstrated that both doping agents preserved an edge-on structure of PBTTT crystallites with similar local ordering. Accordingly, the Seebeck coefficient difference observed when using those dopants was not related to structural changes. The authors linked the enhancement of the Seebeck coefficient in FTS to a more extensive doping of the disordered domains, leading to an increase of the number of percolation pathways[131].

$\mathrm{F}_{4}$ TCNQ is another dopant utilized for PBTTT and depending on the doping process, various properties can be obtained. For example, F 4 TCNQ solution-doped PBTTT films 
demonstrated a power factor of $1.3 \mu \mathrm{W} \cdot \mathrm{m}^{-1} \cdot \mathrm{K}^{-2}$ [132], while vapor-processed PBTTT films with $\mathrm{F}_{4} \mathrm{TCNQ}$ yielded a higher power factor of $32 \mu \mathrm{W} \cdot \mathrm{m}^{-1} \cdot \mathrm{K}^{-2}$ [133]. This increase resulted from a significant difference in the electrical conductivity of the films. Indeed, vapor-doped PBTTT films showed more than hundred times enhancement in electrical conductivity with comparable charge carrier concentrations [132, 133]. This increase in conductivity was attributed to a higher molar ratio of $F_{4} T C N Q$ in the vapor-doped film. PBTTT was also doped with 2,5-difluoro-7,7,8,8-tetracyanoquinodimethane ( $\left.F_{2} T C N Q\right)$, which has a smaller electron affinity than $\mathrm{F}_{4} \mathrm{TCNQ}$. Due to the lower doping efficiency, the vapor-doped film with $\mathrm{F}_{2} \mathrm{TCNQ}$ had a lower conductivity of $36 \mathrm{~S} . \mathrm{cm}^{-1}$ and a higher Seebeck coefficient of $140 \mu \mathrm{V} . \mathrm{K}^{-1}$. Despite the lower doping efficiency, $F_{2}$ TCNQ vapor-doped films had a higher power factor of 70 $\mu \mathrm{W} \cdot \mathrm{m}^{-1} \cdot \mathrm{K}^{-2}$ as compared to the equivalent $\mathrm{F}_{4} \mathrm{TCNQ}$-doped films [133]. The Seebeck coefficient was not strongly morphology-dependent at comparable carrier concentrations. These studies have highlighted the relations between the dopant nature, morphology, and thermoelectric properties in PBTTT and suggest that further improvement can be realized by increasing the charge carrier mobility and controlling the dopant concentration [132-135].

\subsection{Poly [N- 9'-heptadecanyl-2,7-carbazole-alt-5,5-(4,7-di-2-thienyl-} 2',1',3'-benzothiadiazole] (PCDTBT)

PCDTBT was first investigated by the organic photovoltaic (OPV) community to improve the efficiency and stability of OPVs. Recently, the thermoelectric properties of such carbazole-containing copolymers have been investigated as well. For instance, PCDTBT films doped with $\mathrm{FeCl}_{3}$ showed a power factor of $19 \mu \mathrm{W} \cdot \mathrm{m}^{-1} \cdot \mathrm{K}^{-2}$ [136]. Moreover, the influence of the type of carbazole units was investigated by comparing poly((9,9-dioctylfluorene)-2,7diyl-alt-(4,7-bis(3-hexylthien-5-yl)-2,1,3-benzothiadiazole)-2',2"-diyl) (F8TBT) with poly(N-9'heptadecanyl-2,7-carbazole-alt-(4,7-bis(3-hexyl-thien-5-yl)-2,1,3-benzothiadiazole -2',2"diyl) (C8TBT). F8TBT has a fluorene unit as donor, while C8TBT has a carbazole unit. At 373K, the doped C8TBT films had higher electrical conductivity and Seebeck coefficient $\left(0.5 \mathrm{~S} . \mathrm{cm}^{-1}\right.$

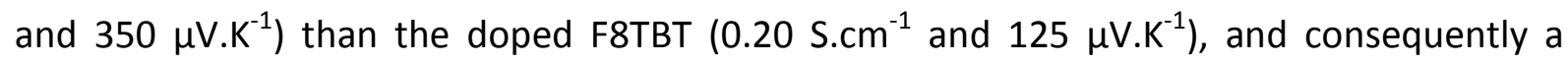
higher power factor of $\sim 12 \mu \mathrm{W} \cdot \mathrm{m}^{-1} \cdot \mathrm{K}^{-2}$ as compared to $~ 0.5 \mu \mathrm{W} \cdot \mathrm{m}^{-1} \cdot \mathrm{K}^{-2}$ [137]. 


\subsection{Poly[2,2'-[(2,5-bis(2-hexyldecyl)-3,6-dioxo-2,3,5,6- tetrahydropyrrolo [3,4-c] pyrrole-1,4-diyl)dithiophene]- 5,5'-diyl-alt- thiophen-2,5-diyl] (PDPP3T)}

PDPP3T is another high-mobility low-band gap semiconductor that is traditionally considered as an excellent $\pi$-conjugated conducting polymer by the organic thin-film transistor community. Recently, PDPP3T films have been evaluated for thermoelectric applications and demonstrated a high PF compared to other thiophene-containing polymers such as P3HT and PBTTT. A comparison of the power factors of Fe(TFSI) ${ }_{3}$-doped PDPP3T, P3HT, and PBTTT showed that PDPP3T has a higher power factor $\left(25 \mu \mathrm{W} \cdot \mathrm{m}^{-1} \cdot \mathrm{K}^{-2}\right)$ than PBTTT (14 $\left.\mu \mathrm{W} \cdot \mathrm{m}^{-1} \cdot \mathrm{K}^{-2}\right)$, and comparable to that of $\mathrm{P} 3 \mathrm{HT}\left(30 \mu \mathrm{W} \cdot \mathrm{m}^{-1} \cdot \mathrm{K}^{-2}\right)$ [131]. It is worth mentioning that PDPP3T films had a lower $\sigma$ and higher $S$ than other films even at low doping levels $[95,131]$. The optimized power factor obtained for PDPP3T films is $276 \mu \mathrm{W} . \mathrm{m}^{-}$ ${ }^{1} \cdot \mathrm{K}^{-2}$. This great performance was attributed to accurate tuning of the doping level with a low volume of dopants that enhanced both the electrical conductivity and the Seebeck coefficient in this high-mobility small-gap semiconducting polymer [138].

\subsection{N-type conductive polymers}

$\mathrm{N}$-type $\pi$-conjugated conductive polymers have been less investigated for thermoelectric devices compared to p-type polymers and the theoretical understanding of the electronic structure and optical transitions towards n-type conducting polymers is still controversial [139]. Moreover, instability in ambient air and low $n$-doping yield have restricted the thermoelectric conversion efficiency of these electron-carrying materials. In general, n-type dopants have low ionization energies to induce an electron transfer to the host polymer which at the same time make them prone to redox reactions with ambient oxygen and moisture, and hence unstable [139]. To avoid this issue, the energy level of their lowest unoccupied molecular orbital (LUMO) must be reduced, for instance, by grafting or inserting electron-withdrawing units in the polymer structure.

There are two common n-doping approaches: (1) using materials that provide direct electron transfer, and (2) using precursor-type dopants. Direct electron transfer occurs via a radical anion to an electron-accepting small molecule; thus, the material shows poor stability when exposed to oxygen and moisture [140]. Whereas precursor-type dopants need an external energy source, e.g. heat or light, to activate the doping agent (cation or anion) 
[141]. The major advantages of precursor-type dopants are easy processability and better stability in ambient conditions. But, miscibility issues may arise and create dopant-rich domains in the material. For the former approach, several electron-carrying small molecules have been investigated, e.g. fullerenes, naphthodithiophenediimide, perylene bisimide, benzotriazole, and naphthalenetetracarboxylic dianhydride [142-146]. For the latter approach, other molecules known for their donor properties like 4-(1,3-dimethyl-2,3dihydro1H-benzoimidazol-2-yl)phenyl)dimethylamine (N-DMBI) [147], tetra-n-butyl ammonium fluoride (TBAF) [148], and cobaltocene $\left(\mathrm{CoCp}_{2}\right)$ [149] have been tested as precursor-type dopants. The development of novel stable dopants exhibiting higher miscibility with n-type polymers is currently extensively investigated [150]. Representative examples of $n$-type materials and dopants are displayed in Figure 5 and several case studies are highlighted in the next sections. 
Figure 5. Chemical structures of common n-type semiconducting polymers and molecules used in thermoelectric applications.

Moses et al. were the first to report $n$-type thermoelectric behavior of polymers in TBAFdoped polyacetylene $\left(S=-43.5 \mu \mathrm{V} \cdot \mathrm{K}^{-1}\right)$ [151]. Following that, $\pi$-conjugated polymers with transition metal atoms in the backbone were shown to behave as $n$-type materials and remain stable in ambient air conditions [152-156]. Currently the best performance reported for an n-type polymer is an organometallic polymer based on metal-ethenetetrathiolate (ETT), poly(Ni-1,1,2,2-ethentetrathiolate) (poly(Ni-ETT)). Indeed, poly((Ni-ETT)-based 
materials processed as a pellet or electrochemically polymerized as a thin film have demonstrated power factors of $66 \mu \mathrm{W} \cdot \mathrm{m}^{-1} \cdot \mathrm{K}^{-2}$ [157] and $344 \mu \mathrm{W} \cdot \mathrm{m}^{-1} \cdot \mathrm{K}^{-2}$ [158], respectively. Other organometals constituted of Ni-S bonds, like copper benzenehexathiol complex (CuBHT), have been investigated and metallic-like properties have been reported with a high $\sigma$ $>1500$ S.cm ${ }^{-1}$ along with a small $\mathrm{S} \sim-4$ to $-10 \mu \mathrm{V} . \mathrm{K}^{-1}$ [159]. Despite these promising results, these materials are known to be hardly processable mainly because of their weak solubility in common solvents. This further hinders their scaling up and industrial applications while limiting their thermoelectric optimization.

Later, Pei et al. investigated p-phenylene vinylene (PPV) substituted with halogen elements like chlorine or fluorine [160]. Accordingly, fluorine functionalized benzodifurandione-based poly(p-phenylene vinylene (FBDPPV) doped with N-DMBI has shown a PF of $28 \mu \mathrm{W} \cdot \mathrm{m}^{-1} \cdot \mathrm{K}^{-2}$ [161]. The thermoelectric properties of conductive polymer films are highly dependent of the morphology, as particularly investigated in p-type polymers. In FBDPPV, Ma and coworkers studied the influence of the dopant concentration on the thin film crystallinity by GIWAXS and optimized formulation yielded to $\sigma=6.2 \mathrm{~S} . \mathrm{cm}^{-1}$, $\mathrm{S}=-210 \mu \mathrm{V} \cdot \mathrm{K}^{-1}$ and $\mathrm{PF}=25.5 \mu \mathrm{W} \cdot \mathrm{m}^{-1} \cdot \mathrm{K}^{-2}$ [162]. Chlorine functionalized benzodifurandionebased poly(p-phenylene vinylene) (CIBDPPV) doped with TBAF also demonstrated good stability in air for a solution-processed n-type polymer where doped films showed $\sigma=0.62$ S.cm ${ }^{-1}$ and PF $=0.63 \mu W \cdot \mathrm{m}^{-1} \cdot \mathrm{K}^{-2}[163]$.

Recently, Lu et al. developed a polymer based on an extended and planar thiophenefused benzodifurandione-based oligo(p-phenylenevinylene) (TBDOPV) and doped it with 4(1,3-dimethyl-2,3-dihydro1H-benzoimidazol-2-yl)phenyl)dimethylamine (N-DMBI-H). This material showed a high degree of planarity with a homogeneous surface morphology,

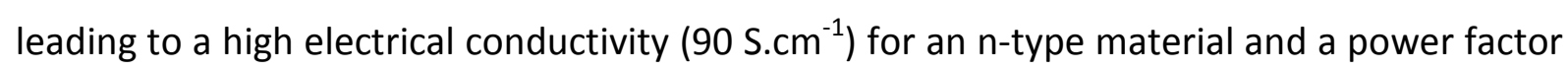
of $106 \mu \mathrm{W} . \mathrm{m}^{-1} \cdot \mathrm{K}^{-2}$. GIWAXS measurements demonstrated that pristine and doped polymers showed a similar $\pi-\pi$ stacking distance while the lamellar spacing distance increased for the doped polymer confirming the miscibility of the dopant. The authors suggested that such high degree of interchain ordering combined with disordered bulk microstructures would be beneficial for the improvement of the carrier mobility [164]. 
Another group of high-performance solution-processable n-type polymers are the naphthalenediimide (NDI) copolymers. Poly[N, $N^{\prime}$-bis(2-octyldodecyl)-1,4,5,8napthalenedicarboximide-2,6-diyl]-alt-5,5-(2,2bithiophene)] P(NDIOD-T2) with the electrondeficient imidazole groups is one of the most famous $n$-type polymers for thermoelectric applications. The doped-PNDIOD-T2 is more stable in ambient air conditions than PPV derivatives. Moreover, they are easier to process as they are soluble in common solvents such as ODCB. Schlitz and coworkers studied PNDIOD-T2 doped with 4-(1,3-dimethyl-2,3dihydro-1H-benzimidazol-2-yl)-N,N-diphenylaniline (N-DPBI) or N-DMBI [165]. The optimization of the doping procedure led to films with a power factor of $0.6 \mu \mathrm{W} \cdot \mathrm{m}^{-1} \cdot \mathrm{K}^{-2}$. Atomic force microscopy (AFM) images showed that N-DMBI molecules strongly aggregated on the films surface, and only $1 \%$ of the dopant molecules contributed to free charge carriers in the films [165]. These results highlight the importance of dopant-polymer miscibility in order to improve the thermoelectric performance of solution-processable ntype polymers. More recent studies have demonstrated improvement of N-DMBI miscibility in PNDIOD-T2 by replacing the NDI alkyl chains with PEG chains [166] or by adding polar side chains to both the NDI and thiophene units [167]. Based on the investigations on PVDIOD-T2, copolymers comprising naphtho[2,3-b:6,7-b]dithiophenediimide (NDTI) and benzobisthiadiazole (BBT) units such as PNDTIBBT-DT or PNDTI-BBT-DP were synthesized and exhibited a $\sigma$ of $5 \mathrm{~S} . \mathrm{cm}^{-1}$ with a power factor of $14 \mu \mathrm{W} \cdot \mathrm{m}^{-1} \cdot \mathrm{K}^{-2}[168]$.

More recently, Koster et al. compared poly(2,2'-bithiazolothienyl-4,4',10,10'tetracarboxydiimide) (PDTzTI) n-type polymer with the PNDIOD-T2 benchmark using TDAE as a dopant [169]. PDTzTI is a thiazole imide-based acceptor-acceptor (A-A) polymer that has recently shown good n-type transport properties. The PF of this n-type polymer at room temperature was measured at $7.6 \mu \mathrm{W} \cdot \mathrm{m}^{-1} \cdot \mathrm{K}^{-2}$ with a figure of merit ZT of 0.01 . The reported power factor is 120 times higher than that of PNDIOD-T2. This improvement was attributed to reduced Coulomb interactions thanks to the large size of the crystallites which increases the distance between the counterions and polymer charges. The A-A character also helps the charge delocalization, which in turn lowers the Coulomb interaction.

Conductive $\pi$-conjugated polymers with a ladder structure are in general soluble in common organic solvents and have been used as n-type thermoelectric polymers. An example of such ladder-type thermoelectric polymers is 
polybenzimidazobenzophenanthroline (BBL) doped with TDAE [170]. Although its power factor is relatively low, $0.43 \mu \mathrm{W} \cdot \mathrm{m}^{-1} \cdot \mathrm{K}^{-2}$, its excellent solubility permits an easy and scalable process similar to p-type PEDOT:PSS.

These studies on doped $\mathrm{n}$-type polymers demonstrated that it is crucial to better understand the doping mechanisms involved in these materials. Additionally, a high miscibility of the dopant in the polymer matrix is primordial in order to enhance their thermoelectric properties. Moreover, their stability in air has to be improved by designing new building blocks which also should provide a good solubility in common solvents.

\subsection{Afterword on $p$ - and n-type polymers}

The room temperature thermoelectric performance of polymeric/organic $p$ - and $n$-type materials are summarized in Figure 6 . The best p-type materials are formulated PEDOT:dopant thin films with 0.2 ? ZT ? 0.4, where the dopants are Tosylate [52] or PSS [83], while the best thermoelectric performance for n-type materials was attested for $\operatorname{poly}(\mathrm{Ni}$ 1,1,2,2-ethenetetrathiolate) thin film with ZT ? 0.2 [158]. The improvement of the thermoelectric properties was mainly performed by the tuning of the doping level. As the electronic properties, i.e. electrical conductivity and Seebeck coefficient are interdependent, Glaudell and co-workers [132] suggested an empirical relation for $p$-type materials: $S$ ? $\sigma^{-1 / 4}$

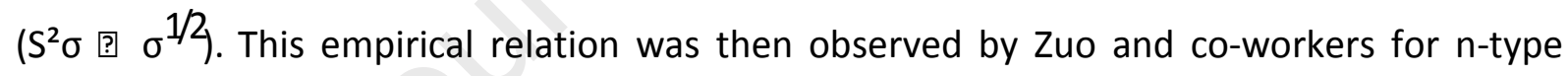
materials [127]. While such generic behavior could not be deciphered using common transport theories, a relatively good approximation has been achieved based on Mott's variable range hopping theory (VRH) with a modified Gaussian density of states by Coulombtrapped ionized dopants. However, this approximation is restricted because of the hypothesis of localized charge carriers [171]. Recently, Lépinoy et al. have demonstrated that this thermopower scaling is a characteristic of 3D Dirac fermions, i.e. massless pseudo relativistic quasiparticles with parabolic density of states, scattered by unscreened ionized impurities, lifting the restriction and opening the door of Dirac formalism in $\pi$-conjugated polymer materials [172]. The light grey lines in Figure 6 represent a constant ZT estimated assuming a phonon contribution to the thermal conductivity of $\mathrm{kph}=0.2 \mathrm{~W} \cdot \mathrm{m}^{-1} \cdot \mathrm{K}^{-1}$ [173]. However, the correlation between the electronic contribution to the thermal conductivity $\mathrm{K} d$ and the electrical conductivity, inherent to the Wiedemann-Franz law, increases the total thermal conductivity $(\kappa=\kappa \mathrm{ph}+\mathrm{kel})$, inhibiting further improvement of the figure of merit $\mathrm{ZT}$. 
One possible strategy to bypass this limitation is the decoupling of the electronic and thermal conductivity by the formation of heterogeneous materials, i.e. composites of conductive polymers with inorganic materials in order to enhance phonon scattering.

Figure 6. Thermoelectric power factor as function of electrical conductivity for p-type (blue: polythiophene derivates, purple: PEDOT, green: polyacetylene) and n-type materials extracted from literature. The dashed lines represent the relation $S$ ? $\sigma^{-1 / 4}$. The gray lines represent lines of a constant figure of merit ZT [173].

\section{Polymer composite thermoelectric m aterials}

To date, most of the investigations on polymer thermoelectric compounds have targeted the enhancement of their electrical conductivity. Conversely, in the case of inorganic materials, efforts have been directed towards the reduction of the thermal conductivity of optimally doped systems. The key difficulty in optimizing these properties is the strong antagonistic correlation of $S, \sigma$, and $k$ in most systems. It is in this scenario that polymer thermoelectric materials hybridized with inorganic nanomaterials have gained momentum, aiming at the synergistic combination of low thermal conductivity of polymers and the high Seebeck coefficient of the inorganic semiconductors. The interactions between the nanofillers and the polymer matrix can indeed yield the decoupling of the thermoelectric parameters leading to an increase of the power factor with respect to the sole constituents. Thermoelectric composite fillers can be either inorganic or organic while a range of matrices with different electrical conductivities can be employed $[155,174]$. 


\subsection{Polymer-polymer composites}

A known strategy aiming to minimize the thermal conductivity of thermoelectric composites is the use of a nanostructured second phase embedded in a conductive polymer matrix. Alternatively, blending of two different conductive polymers with different HOMO levels has been shown to create new states close to the Fermi level [18]. Such band structure engineering is highly relevant since the resulting modification of the density of states and its slope at $\varepsilon F$ can induce an increase of the electrical conductivity and the Seebeck coefficient values, respectively.

One of the most popular nanostructured thermoelectric composites is made from PEDOT nanowires embedded in a PEDOT:PSS matrix. By adding a small amount of PEDOT nanowires

(0.2 wt\%), these composites have demonstrated a high power factor of $446.6 \mu \mathrm{W} \cdot \mathrm{m}^{-1} \cdot \mathrm{K}^{-2}$ with a Seebeck coefficient of $59.3 \mu \mathrm{V} . \mathrm{K}^{-1}$ and an electrical conductivity of $1270{\mathrm{~S} . \mathrm{cm}^{-1}}^{-1}$ (i.e. ZT

0.44 at room temperature) [175]. This high PF was attributed to an interfacial energy filtering effect. The interfaces between the two components induce an energy-dependent charge carrier scattering by setting potential barriers or traps that filter low energy charge carriers. This results in the enhancement of the Seebeck coefficient without detrimental effects on the electrical conductivity. A similar strategy has been reported for blends of P3HT with PTB7 or TQ1 for which a record value of the Seebeck coefficient $\sim 1 \mathrm{mV} . \mathrm{K}^{-1}$ at room temperature was reported [176].

\subsection{Polymer-inorganic composites}

Producing polymer-inorganic composites for thermoelectric applications is one of the main strategies to improve the performance of already well-known inorganic materials by lowering their thermal conductivity without significantly affecting other properties. Polymer matrices may also provide some degree of flexibility and lower volumetric cost. On the other hand, producing polymer-inorganic composites for thermoelectric applications is another strategy to improve the performance of already well-known inorganic materials by lowering their thermal conductivity without significantly affecting other properties. Polymer matrices may also provide some degree of flexibility and lower volumetric cost. Nevertheless, the maximum operational temperature is subsequently limited to values typically lower than $500 \mathrm{~K}$ 
The first attempt to produce polymer-inorganic composites for thermoelectric applications was performed by filling an array of vertical silicon nanowires by an insulating polymer in order to both decrease the total $\mathrm{k}$ and benefit from an increase in $\mathrm{S}$ due to the reduced dimensionality of the nanowires [177]. However, a strong decrease of $\sigma$ was noticed following this strategy. In order to prevent such effect, Zhang et al. used a conductive PEDOT:PSS as matrix hybridized with ball-milled bismuth telluride $\left(\mathrm{Bi}_{2} \mathrm{Te}_{3}\right)$. Power factors of 131 and $80 \mu \mathrm{W} \cdot \mathrm{m}^{-1} \cdot \mathrm{K}^{-2}$ for $\mathrm{p}$-type and n-type composites, respectively, were reported at room temperature, highlighting the potential of this approach. However, the PF values remained low as compared to the bulk $\mathrm{Bi}_{2} \mathrm{Te}_{3}$ performance due to the relatively high contact resistance between the constituents.

In order to better apprehend the thermoelectric behavior of polymer-inorganic material composites, $\mathrm{He}$ et al. performed a systematic study on $\mathrm{P} 3 \mathrm{HT}$ and $\mathrm{Bi}_{2} \mathrm{Te}_{3}$ nanowires composites. For such samples, an optimal PF value of $13.6 \mu \mathrm{W} \cdot \mathrm{m}^{-1} \cdot \mathrm{K}^{-2}$ at $300 \mathrm{~K}$ (three times larger than pristine $\mathrm{P} 3 \mathrm{HT}$ ) was recorded due to an energy-dependent carrier scattering mechanism [178]. Indeed, while energy-filtering at interfaces induces a decrease of the electrical conductivity due to the localization of low energy charge carriers, the introduction of additional heterojunction interfaces coupled with optimized charge carrier concentration and mobility leads to an increase of the Seebeck coefficient. Another study on composites made from Te nanowires, PEDOT:PSS and reduced graphene further confirmed the benefit of the energy-filtering effect by achieving a power factor of $143 \mu \mathrm{W} \cdot \mathrm{m}^{-1} \cdot \mathrm{K}^{-2}$ at $300 \mathrm{~K}$, which is 1-2 orders of magnitude higher than those of the single- or binary-component materials [179].

Conversely, Liang et al. stated that the energy-filtering effect does not necessarily occur in nanocomposites. By carefully controlling the amount of $\mathrm{FeCl}_{3}$ dopant in the polymer phase, they could tune the energy barrier between P3HT and TE nanowire composite close to $1 \mathrm{eV}$. Although the composites' PF increased beyond those of the pure polymer and nanowires, the authors concluded that the energy-filtering effect was not necessary to explain the enhanced Seebeck coefficients and PFs. Instead, simple two materials transport models in parallel, in series as well as the linear combination of both models could yield similar results in the composites [180]. 
Another research strand in polymer-inorganic composites has focused on the thermal conductivity. Conventional inorganic thermoelectric materials are characterized by high thermal conductivities of $1.2-2.8 \mathrm{~W} \cdot \mathrm{m}^{-1} \cdot \mathrm{K}^{-1}[180,181]$, almost ten times higher than the ones reported for polymeric materials [182]. In polymer-inorganic composites, an important phonon-scattering rate is induced by the large number of interfaces, resulting in composites with thermal conductivities close to those of the polymer matrices, e.g. $\mathrm{K}=0.3 \mathrm{~W} \cdot \mathrm{m}^{-1} \cdot \mathrm{K}^{-1}$ for PEDOT:PSS with 0.8 wt\% Te nanowires and $\mathrm{k}=0.4 \mathrm{~W} \cdot \mathrm{m}^{-1} \cdot \mathrm{K}^{-1}$ for PEDOT:PSS with $0.5 \mathrm{wt} \%$ SnSe nanosheets $[126,127,183-185]$.

The best figure of merit for p-type composites with a ZT of 0.58 at room temperature has been obtained for a PEDOT- $\mathrm{Bi}_{2} \mathrm{Te}_{3}$ nanocomposite. The in-situ polymerization of PEDOT in presence of $\mathrm{Bi}_{2} \mathrm{Te}_{3}$ nanoparticles allowed an intimate contact between both materials, enhancing the charge transport across the interfaces [186]. For n-type composites, Zhao et al. have recently reported a hybrid of PVDF and Ni nanowires which exhibits an electrical conductivity of $4701 \mathrm{~S} . \mathrm{cm}^{-1}$ and a Seebeck coefficient of $-20 \mu \mathrm{V} . \mathrm{K}^{-1}$, resulting in a power factor of $200 \mu \mathrm{W} \cdot \mathrm{m}^{-1} \cdot \mathrm{K}^{-2}[187]$.

\subsection{Polymer-nanocarbon composites}

Nanostructured carbon allotropes, like graphene and carbon nanotube (CNT), have been considered as filler elements for thermoelectric composite materials in order to benefit from the intrinsic high electrical conductivity [70, 188-190]. Similar to polymer-inorganic thermoelectric composites, the nanocarbon-based thermoelectric composites have demonstrated the possible decoupling between the electronic transport properties, $\mathrm{S}$ and $\sigma$. Furthermore, the thermal conductivity of these composites is low due to extensive phonon scattering at the interfaces.

At first sight, graphene may not be considered as a promising candidate for thermoelectric applications due to its symmetric density of states and high thermal conductivity. Nevertheless, Du et al. produced composites made from PANI and graphene nanosheets and reported a relatively good power factor of $5.6 \mu \mathrm{W} \cdot \mathrm{m}^{-1} \cdot \mathrm{K}^{-2}$ due to the high charge carrier mobility of the composite [183]. Wang and co-workers used a mixture of graphene, $C_{60}$ and PEDOT:PSS to obtain a higher power factor of $32 \mu \mathrm{W} \cdot \mathrm{m}^{-1} \cdot \mathrm{K}^{-2}$ and a $Z T$ of 0.067 at $300 \mathrm{~K}$ [191]. Another approach utilizing graphene is based on a layer-by-layer 
assembly of stabilized CNT and graphene with PANI which has demonstrated a PF of 1825 $\mu \mathrm{W} . \mathrm{m}^{-1} \cdot \mathrm{K}^{-2}$ at $300 \mathrm{~K}$ [192]. Such a high PF was achieved thanks to an enhanced charge carrier mobility and bridge-like interconnections between the multilayers provided by the CNTs. The same layer-by-layer assembly was applied to the production of an n-type composite with polyethyleneimine and graphene, and a PF of $190 \mu \mathrm{W} \cdot \mathrm{m}^{-1} \cdot \mathrm{K}^{-2}$ was measured at room temperature [193].

CNTs are one of the most popular nanofillers for thermoelectric applications. PolymerCNT thermoelectric composites can be categorized based on the following basic principles: electronic percolation, secondary doping (tuning of the charge carrier concentration and mobility) and energy-filtering. One of the first reports using CNTs was based on a composite with PEDOT:PSS embedded in an Arabic gum matrix and demonstrated that the electrical conductivity could be partially decoupled from the thermal conductivity [194, 195]. The underlying mechanism is related to the creation of percolative conductive paths for electrons, while heat flows in parallel between the insulating matrix and small amounts of the conductive filler.

Moriarty et al. prepared a suspension of PEDOT:PSS particles and single-wall CNTs (Figure 7(a)) and managed to obtain water-processable and flexible thin films with high electrical conductivities of $4000 \mathrm{~S} . \mathrm{cm}^{-1}$ and low thermal conductivities of $0.4-0.7 \mathrm{~W} \cdot \mathrm{m}^{-1} \cdot \mathrm{K}^{-1}$. The high $\sigma$ was inherent to the creation of electrical junctions between the percolated CNTs (Figure $7(b, c))$. A power factor of $140 \mu \mathrm{W} \cdot \mathrm{m}^{-1} \cdot \mathrm{K}^{-2}$ was achieved at $300 \mathrm{~K}$ for thin films composed of 85 wt\% CNTs [196]. 

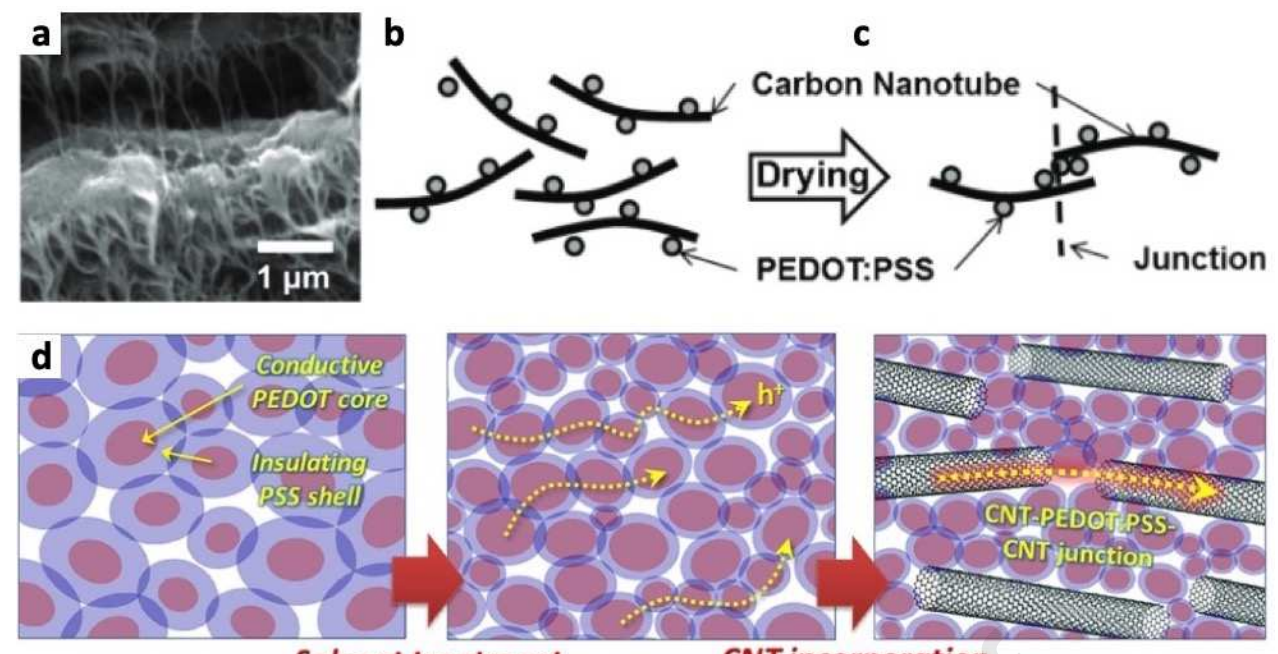

Solvent treatment

CNT incorporation
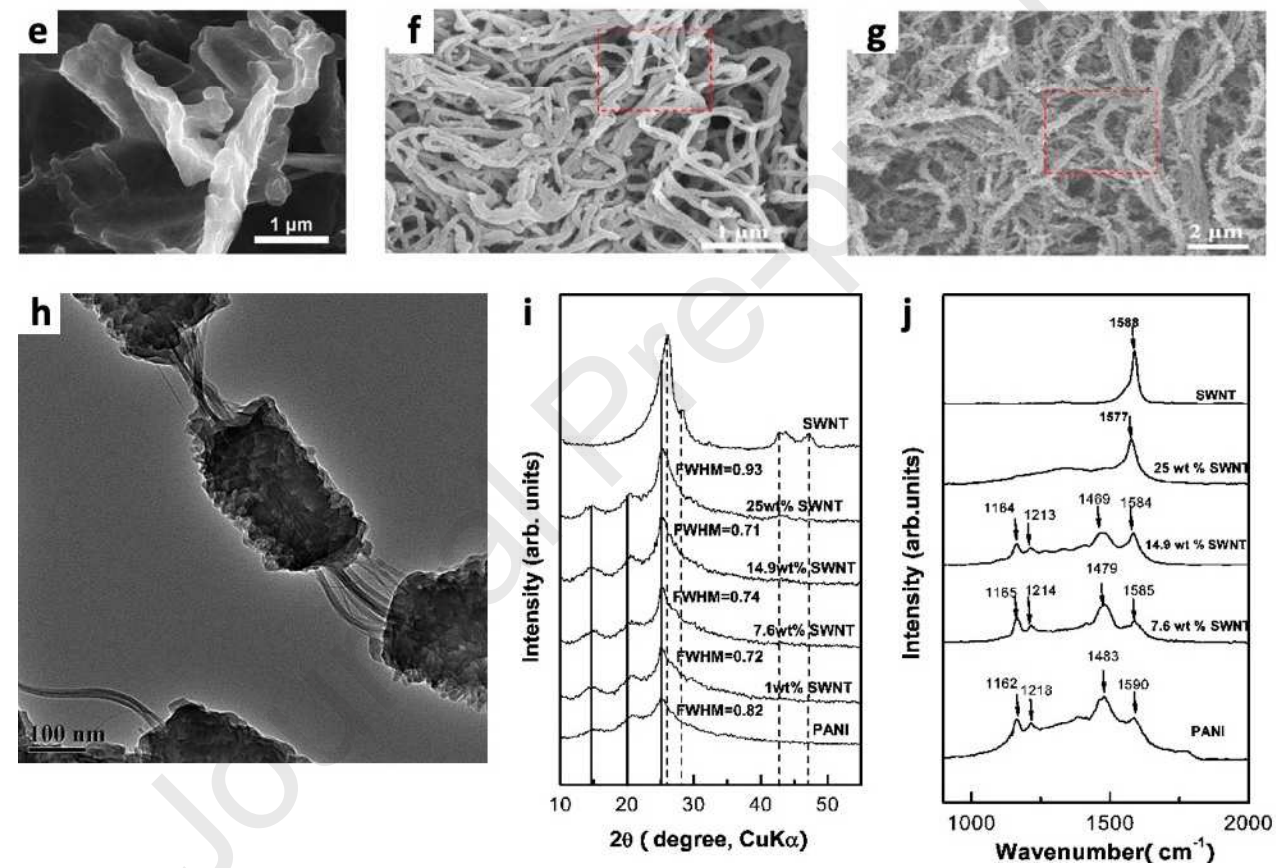

Figure 7. (a) SEM image of PEDOT:PSS-SWCNT composite films. (b, c) Schematics of CNTs coated by PEDOT:PSS particles in suspension and after drying from the exfoliated state [195]. (d) Conceptual illustrations of solvent treatment and CNT incorporation effects [197]. (e) SEM image of PEDOT-rGO oxide [198]. (f, g) SEM images of PEDOT-SWCNT composites displaying cable-like and coral-like morphologies, respectively [199]. (h) SEM image of a SWCNT-PANI composite with $25 \mathrm{wt} \%$ SWCNT. (i) XRD patterns of SWCNT-PANI composites with different SWCNT content (solid and dashed lines denote the peaks of PANI and SWCNT, respectively). (j) Raman spectra for SWCNT-PANI composites with different SWCNT content [200].

Post-treatments were used to further improve the thermoelectric properties of composites based on CNTs. For example, formic acid and DMSO were used for treating CNTPEDOT:PSS composites in order to enhance the electrical conductivity of the composites [197]. The solvent treatment induces a better percolation between the PEDOT conductive 
islands by removing excess PSS while the addition of CNTs allows the formation of CNT- 


\begin{tabular}{|c|c|c|c|c|c|c|c|c|c|}
\hline PPV:I & 313 & - & 34 & 183.5 & 43.5 & 0.80 & 34.7 & $1.36 \times 10^{-2}$ & {$[49]$} \\
PEDOT:PSS (EG
\end{tabular}

+DMSO) 


\begin{tabular}{|c|c|c|c|c|c|c|c|c|c|}
\hline $\begin{array}{c}\text { PEDOT:PSS + } \\
\text { PEDOT nanowires }\end{array}$ & RT & - & - & 1270 & 59.3 & 1.1 & 446.6 & 0.44 & {$[175]$} \\
\hline $\begin{array}{c}\text { PEDOT:PSS + } \\
\mathrm{Bi}_{2} \mathrm{Te}_{3}\end{array}$ & RT & - & $23 \mathrm{wt} \%$ & 300 & 30 & 0.47 & 119 & 0.06 & {$[181]$} \\
\hline
\end{tabular}

PEDOT:PSS + Te

nanowires, and

reduced

graphene 

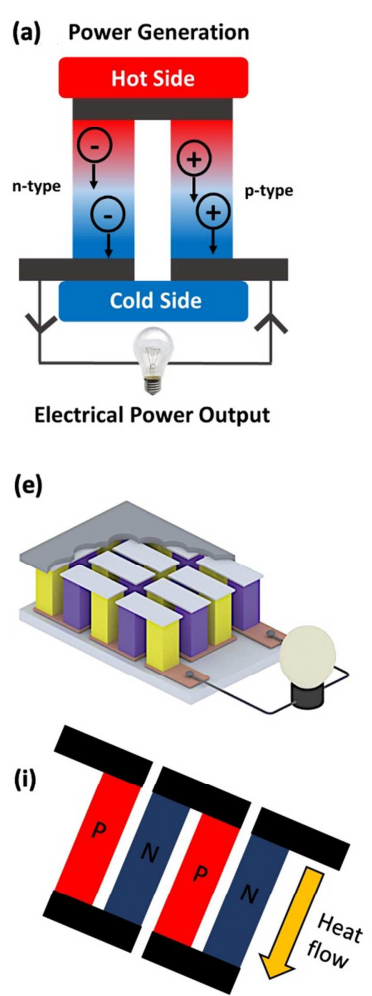
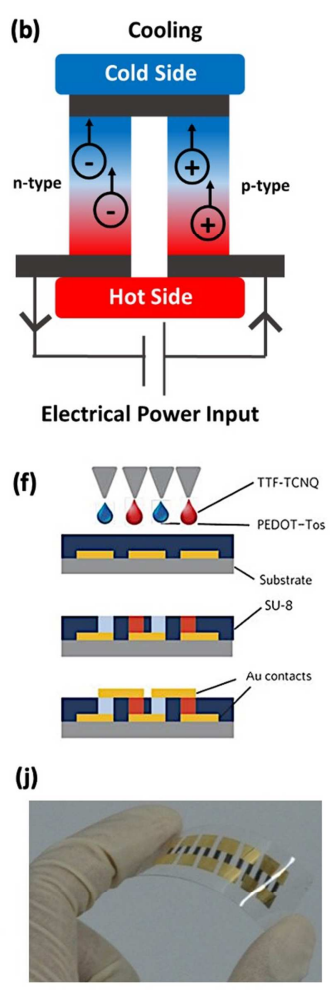

(c)
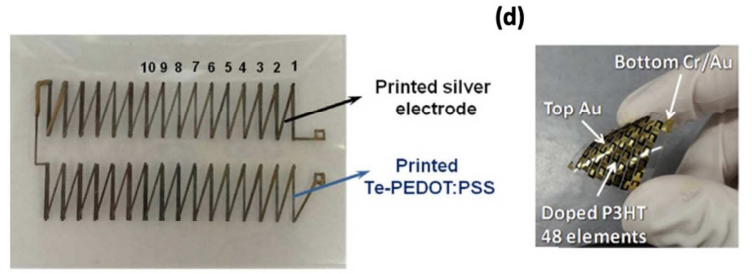

(g)

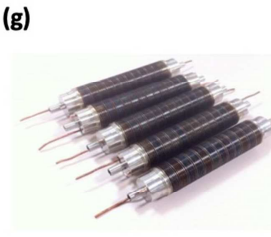

(h)
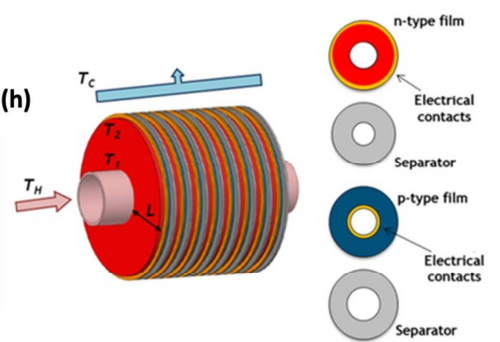

(k)

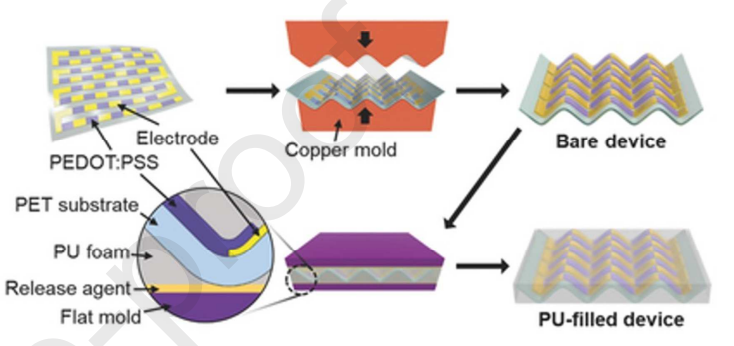

Figure 8. (a, b) Schematic representations of Seebeck and Peltier effects used for thermoelectric power generation and cooling, respectively. (c) Image of a 32-legs planar thermoelectric generator on a flexible PET substrate [62]. (d) Image of a 48-element 3D thermoelectric generator on a flexible PET substrate [205]. (e) Schematic representation of a TEG with flat bulk square legs [206]. (f) Manufacturing process used to fabricate an organic flat bulk TEG [52]. (g) Cylindrical TEGs developed by Gentherm [207]. (h) Schematic representation of a TEG with a radial geometry [208-210]. (i) A schematic of a thin film TEG consisting of deposited $\mathrm{p} / \mathrm{n}$ legs and connectors. (j) An organic thin film " $\pi$ structure" TEG, applicable when heat propagates in-plane [211]. (k) Schematic description of the fabrication process of a chevron-structured TEG [212].

The conversion of heat to electricity is useful for various industrial sectors, such as electronics, petroleum industry, automotive industry, renewable energy, medical sensing, etc. In fact, TEGs can be used with every technology that deals with heat management. However, low ZT and feeble conversion efficiency are the main drawbacks of thermoelectric systems. In recent years TEGs have gained increasing momentum as evidenced by their market growth from $\$ 40$ million in 2014 to $\$ 90$ million in 2016. Highlighting this growth, IDTechEx has anticipated a \$1 billion market for TEGs by 2026 mainly arising from innovative applications in vehicles' waste heat recovery systems, wireless sensor networks, and customizable consumer applications under Internet of Things (IOT) [213].

IoT, as a great enabler, is foreseen to significantly impact the technology market, various industries and our society as a whole. It is estimated that wirelessly connected sensors to 
devices and objects will provide permanent communication through sophisticated ubiquitous networks, thus generating continuous data flow (knows as Big Data) and energy consumption. In 2020, more than 25 billion devices were estimated to be connected following such concepts [214], each requiring an energy source and generating waste heat concomitantly. Therefore, TEGs are probably the best technology to solve this problem by harnessing the residual heat and producing electricity. In this regard, compared to inorganic materials, organic and polymer-based TEGs have several benefits, like flexibility and size adaptability, cheap materials and manufacturing processes, environmentally friendly components, and no maintenance requirements. In fact, loT can propel organic and polymeric thermoelectrics from niche to market.

One of the major assets of polymeric TEGs is their printability, flexibility, and mouldability $[16,43]$. Printing techniques allow large scale operation and low-cost production of numerous thermoelectric legs with the aim to generate high thermo-voltage per device area in a sole manufacturing process on flexible substrates. As a result, printable polymeric TEGs are excellent candidates for use in self-powered devices. For example, Figure 8(c) shows 2D printed thermoelectric arrays of silver and tellurium-PEDOT:PSS legs that could generate a stable thermo-voltage of over $2 \mathrm{mV}$ in response to human body heat [62]. Figure $8(\mathrm{~d})$ shows a flexible 3D drop cast TEG made of 24 pairs of $p$ and $n$-legs that reached an output power of $\sim 2.5 \mu \mathrm{W}$ at $\Delta \mathrm{T} \sim 35 \mathrm{~K}[205]$.

\subsubsection{TEGs architecture}

Depending on the heat source surface shape, various TEG architectures have been developed. The main challenge of any TEG architecture is to conserve a good thermal gradient while minimizing heat loss, thermal contact resistances, and device cost.

\subsubsection{Flat bulk TEGs}

Inorganic TEG modules are commonly built in a flat bulk architecture [206]. The flat bulk TEGs constitute of cuboid legs in an alternating p-type and n-type arrangement (Figure 8(e)), through which electrical and thermal currents run simultaneously. An experiment using inorganic flat bulk TEGs in the automotive industry produced up to $1 \mathrm{~kW}$ from exhaust gas heat [215]. In 2011, the first polymer-based thermoelectric device using flat bulk architecture was developed by Bubnova et al. in which, PEDOT:Tos (p-type) and TTF-TCNQ 
(n-type) were used as thermoelectric materials and processed by ink-jet printing, as schematically shown in Figure 8(f). This bulk configuration reached an output power of 0.128 $\mu \mathrm{W}$ with a gradient of $10 \mathrm{~K}$ at $300 \mathrm{~K}$ [52].

\subsubsection{Radial bulk TEGs}

Another bulk TEG design is the cylindrical shape that constitutes of ring-shaped legs. This shape is the most appropriate to harvest electricity from cylindrical objects, like pipelines or automobile exhaust pipes. In 2015, Gentherm company developed cylindrical inorganic TEG prototypes to boost vehicle power efficiency (Figure 8(g)). A 30 minute driving experiment produced an average output power of $\sim 30 \mathrm{~W}$ for an individual TEG module where the exhaust gas temperature reached up to $\sim 600^{\circ} \mathrm{C}$ [207]. A similar architecture was adopted by Menon et al. (Figure $8(\mathrm{~h})$ ) to place their TEG around a warm fluid pipe and their devices operate without addition of heat spreaders [208-210]. This TEG configuration produced a 85 $\mathrm{mV}$ open circuit voltage and a power density of $15 \mathrm{nW} . \mathrm{cm}^{2}$ at $\Delta \mathrm{T}=50 \mathrm{~K}$.

\subsubsection{Thin film TEGs}

The development of low cost and straight forward manufacturing techniques, such as printing technology, has allowed freedom of design for TEGs architecture. It is now possible to go beyond the bulk geometries and develop TEGs in thin layers. Contrary to inorganic TEGs made of thick bulk legs, solution-processable organic thermoelectric materials can be cast in unconventional geometries such as the " $\pi$ structure", as schematically shown in Figure $8(\mathrm{j})$. Controlled deposition of thermoelectric materials as thin layers enables the manufacturing of two-dimensional devices for applications where the heat is applied inplane and propagates along the layers. An example of an inkjet-printed thin film TEG is shown in Figure 8(i), where a thermo-voltage of $15 \mathrm{mV}$ was obtained with $\Delta \mathrm{T}=25 \mathrm{~K}$ [211].

\subsubsection{Flexible polymer-based TEGs}

The first polymer-based thermoelectric device was made of PEDOT:Tos ( $p$-type) and TTFTCNQ (n-type) in a bulk head-to-head configuration. Using this organic TEG, Bubnova et al. could obtain a $0.128 \mu \mathrm{W}$ power at room temperature with $\Delta \mathrm{T}=10 \mathrm{~K}$ [52]. Because of the poor stability and efficiency of n-type polymer, most reported devices so far have used only p-type polymers interconnected in series. As expected, such devices show lower performance than regular $\mathrm{p}$-type/n-type configuration; however, by controlling the leg 
numbers and the device architecture (e.g. connection between legs in series head-to-tail), comparable results can be obtained. Based on this model, screen printed TEGs on paper made of PEDOT:PSS and silver connections demonstrated a power output of $4 \mu \mathrm{W}$ with $\Delta \mathrm{T}=$ $50 \mathrm{~K}$ [216]. The same architecture and materials were used in a thermal lamination method to produce a polymeric TEG which exhibited a power output of $37 \mu \mathrm{W}$ with $\Delta \mathrm{T}=50 \mathrm{~K}$ at 300 $K$ [217]. The influence of the device architecture was investigated by comparing the power output of 3D (drop-cast, thick film) and 2D (spin-coated, thin film) devices [218]. The 3D TEG demonstrated up to 7 times higher power, $175 \mathrm{nW}$, with a temperature gradient of $50 \mathrm{~K}$, than the 2D TEG. Later, Kim et al. developed a flexible organic TEG with PEDOT:PSS in a chevron-structure (Figure 8(k)) [219]. They managed to integrate 24 PEDOT:PSS legs onto a flexible substrate and obtain a power output of $1 \mu \mathrm{W}$ at $\Delta \mathrm{T}=17.5 \mathrm{~K}$ with a heat flow perpendicular to the device.

The optimization of the device architecture is also crucial to improve the power output of TEGs. Particularly in the case of small flexible TEGs, the short leg lengths allow a limited temperature gradient across the active materials. Therefore, to enhance further the thermovoltage, a larger number of $p$ - and n-type leg pairs is mandatory. However, Gordiz et al. suggested that too many thermoelectric legs with relatively large spacings between them could induce a high interconnect resistance, and could limit the device performance [208]. To overcome this problem and obtain a higher output power, they positioned the legs in a hexagonal close-packed pattern which leads to higher fill factors and lower interconnect resistance as compared to circular and square cross-sectioned thermoelectric legs.

Menon et al. tested a thermoelectric device with a radial architecture that produced an $85 \mathrm{mV}$ open circuit voltage and a power density of $15 \mathrm{nW} . \mathrm{cm}^{-2}$ at $\Delta \mathrm{T}=50 \mathrm{~K}$ under natural heat convection $[208,210]$. Later, PEDOT:PSS was coated on commercial polyester fabrics [220] and on silk yarn [221]. These wearable polymer-based TEGs generated a maximum power of $388.7 \mu \mathrm{W}$ at $\Delta \mathrm{T}=75 \mathrm{~K}$ with two strips and of $12.3 \mathrm{nW}$ at $\Delta \mathrm{T}=66 \mathrm{~K}$ with 26 legs on a commercial fabric and silk yarn, respectively. Free standing PEDOT:PSS films were also evaluated by Li et al. and they demonstrated a high electrical conductivity $\left(2500 \mathrm{~S} . \mathrm{cm}^{-1}\right)$ derived from the improved stacking of polymer chains in this configuration. They demonstrated a 5-leg module with a thermo-voltage of $2 \mathrm{mV}$ at $\Delta \mathrm{T}=25 \mathrm{~K} \mathrm{[222].}$ 
Furthermore, photolithography has been used to manufacture flexible organic TEGs [223]. Satoh et al. used PEDOT:PSS and TTF-TCNQ as p-type and n-type materials to manufacture thermoelectric legs for a $\pi$-type module structure. An output voltage of $250 \mathrm{mV}$ was obtained at $\Delta T=80 \mathrm{~K}$, which was enough to power selected devices, even if the addition of a booster circuit was required. However, high contact resistances, detrimental to the overall efficiency, were observed, and the authors underlined the needs to design materials from the viewpoints of device architecture and large-scale manufacturing rather than only focusing on the optimization of the materials for higher figures of merit (ZT) [223].

\subsubsection{Flexible polymer-inorganic composite TEGs}

Various TEGs have been made using polymer-inorganic composite materials, aiming to combine the polymer's flexibility and inorganic material's thermoelectric performance. Dong et al. prepared flexible and transparent TEGs on polyethylene terephthalate (PET) substrates. Designing a device of 8 leg pairs composed of PEDOT:PSS and a hybrid indium tin oxide (ITO)-PEDOT:PSS as $\mathrm{p}$ - and n-type materials, respectively, they recorded a voltage output of $6.8 \mathrm{mV}$ and power output of $0.86 \mathrm{nW}$ under a thermal gradient of $20 \mathrm{~K}$. Despite the composite nature of the n-type material, no improvement of the thermoelectric properties was observed, revealing the domination of ITO. However, it was proposed that depositing PEDOT:PSS on top of ITO enhances the mechanical stability to bending [224].

Ferhat et al. made an entirely inkjet-printed flexible TEG on paper (Figure 9(a)). They used PEDOT:PSS as p-type and (TiS2 2 HA $)_{x}$ ) as n-type legs, and obtained a power output of $22.5 \mathrm{nW}$ at $\Delta T=20 \mathrm{~K}$ for a small device surface of $128 \mathrm{~mm}^{2}$ [225]. Tian et al. designed a $5 \mathrm{p}$-n pairs device constituted of hybrid TiSz-organic superlattice film and PEDOT:PSS film, as $n$ - and $p$ type legs, respectively. They recorded a voltage output of $33 \mathrm{mV}$ along with a maximum power density of $250 \mu \mathrm{W} . \mathrm{cm}^{-2}$ with $\Delta \mathrm{T}=70 \mathrm{~K}$ [226]. Fang et al. screen printed a rolled flexible TEG with PEDOT:PSS and conjugated polyelectrolytes with nickel (CPE)/CNT nanocomposite as p-type and n-type legs, respectively [204]. From a TEG with 288 legs, a maximum output power of $46 \mu \mathrm{W}$ and open circuit voltage of $260 \mathrm{mV}$ was measured at $\Delta \mathrm{T}=$ $65 \mathrm{~K}$ (Figure 9(b)). 
Figure 9. (a) Flexible TiS2 $(\mathrm{HA})_{X}$ nanocomposite TEG printed on paper [225]. (b) Fabrication process for the rolled TEGs using PEDOT:PSS as p-type and CPE/CNT nanocomposite as n-type legs [204]. (c) Schematic illustration of assembling a PEDOT-based TEG [227]. (d) Architecture of an all-fabric thermopile [228].

\subsubsection{Polym er-based wearable TEGs}

One of the most promising applications for polymer-based TEGs is in self-powered wearable electronic devices, as TEGs can directly convert human body heat into electricity required to power these appliances. It is theoretically predicted that a power of $5 \mathrm{~mW}$ could be harvested from human body in an indoor environment [229]. The most important features for wearable devices in general and wearable TEGs in particular are light weight, biocompatibility, flexibility, and mechanical robustness. Conductive polymer-based materials can perfectly match these requirements. A familiar example of wearable TEGs used for harvesting body heat is self-powered watches. However, the surface area of such wearable TEGs is very small compared to the total human body surface area [230, 231]. Hence, research has been directed toward embedding TEGs into textile to harvest the heat from a much larger surface area.

Such electronic textiles need to be fabricated from durable and electrically conducting yarns. Ryan et al. developed a scalable fabric-dyeing process by soaking silk in a PEDOT:PSS bath [221, 232]. Using such a process, a flexible, air-permeable, fabric-TEG was made by soaking a commercial fabric in a PEDOT:PSS solution. The product demonstrated a stable thermoelectric performance between $300 \mathrm{~K}$ and $390 \mathrm{~K}$, and a high voltage output of $4.3 \mathrm{mV}$ at $\Delta T=75.2 \mathrm{~K}$ [220]. However, textile processing with polymeric yarns is challenging, 
because the applied strain and weak adhesion of polymers to the yarns (poor wettability) can lead to exfoliation and high electrical resistance [212]. That being said, there have been new approaches such as vapor phase polymerization (VPP) that can result in uniform polymer coatings on textile $[233,234]$.

Jia et al. developed multi-layered PEDOT-coated electronic textiles using such in situ VPP method (Figure 9(c)) and measured an output voltage of $5 \mathrm{mV}$ at $\Delta \mathrm{T}=25 \mathrm{~K}$ [227]. Similar VPP process was used to produce an all-fabric thermopile from PEDOT-based ink on commercial cotton (Figure 9 (d)) [228]. Alison et al. showed that the reactive vapor coating process can create mechanically rugged conductive coatings that lead to high efficiency thermopiles. The reported vapor-coated cotton fabric has the highest thermoelectric efficiency of any $\mathrm{p}$-type conducting fabric to date, with a Seebeck coefficient of $16 \mu \mathrm{V} . \mathrm{K}^{-1}$ and a power factor of $0.48 \mu \mathrm{W} \mathrm{m}{ }^{-1} \cdot \mathrm{K}^{-2}$ at $\Delta \mathrm{T}=75 \mathrm{~K}$ [220]. The thermopile was then integrated into a wearable band that generated a thermovoltage of $23 \mathrm{mV}$ when worn on the hand.

\subsection{Polymer-based thermoelectric coolers (TECS)}

The use of conductive polymers for TECs is still in its infancy. Hu et al. developed a proof of concept cooling fabric made of conductive polymer material by immerging a commercial textile in an aqueous solution containing pyrrole and ferric chloride [235]. The authors observed a small temperature difference of $1 \mathrm{~K}$ while testing the sample with a Peltier test rig. However, this temperature difference gradually diminished during the experiment because of the polymer degradation and the poor thermal insulation. This preliminary result demonstrated the possibility to design a polymer-based cooling fabric. More recently, Jin et al. investigated the Peltier effect in a poly(Ni-ett) film and measured a maximum $\Delta \mathrm{T}$ of $38 \pm 3$ $\mathrm{K}$ while applying a current density of $5 \mathrm{~A} \cdot \mathrm{mm}^{-2}$ [236]. These results suggest that polymers are promising candidates also for cooling purposes. However, compared to commercialized Peltier modules made of $\mathrm{BiTe} / \mathrm{PbTe}$ (that can create up to $75 \mathrm{~K}$ temperature difference), polymer-based TECs still need further developments and additional fundamental breakthroughs are needed to improve their efficiency and reliability. 


\section{Conclusion and outlook}

Since the discovery of the thermoelectric effect at the end of the $18^{\text {th }}$ century, novel developments towards the integration of thermoelectricity into practical devices for waste heat management have blossomed. Progress in semiconductors processing in the 1950's allowed the commercial exploitation of thermoelectric generators and cooling modules in niche markets with high-added value. In fact, the simple working principles of thermoelectric devices combined with the absence of noise and moving parts, which allow the pollutionfree conversion of a temperature gradient to electricity (and vice versa), make them one of the most robust energy conversion systems, as evidenced by the radioisotope-TEGs onboard of Voyager I and II space probes sent to space in 1977.

However, thermoelectric research was reinvigorated in the past two decades by the development of novel microfabrication techniques to produce smaller, cheaper, and more efficient thermoelectric devices. Nowadays TEGs can be produced in millimeter size yet include hundreds of thermocouples to generate considerable amounts of electricity from temperature differences of only a few degrees. The frontiers of thermoelectric research are being pushed further on by using inorganic nanomaterials and more recently organic and polymeric materials. Nanotechnology provides a path to separate a material's electric and thermal properties which should leverage a substantial improvement of the thermoelectric figure of merit. In parallel, flexible thermoelectric devices and thermoelectric fabrics made of organic and conducting polymers are being widely studied with the aim to use them in body energy harvesters directly integrated into our clothing. The produced electricity can subsequently power wearable sensors and actuators embedded in the garment.

Nevertheless, the thermoelectric polymer field is still in its infancy with respect to the well-established inorganic thermoelectric domain. While semiconducting polymers benefit from better flexibility and absence of toxic elements, compared to inorganic semiconductors such as the bismuth telluride alloy family, their overall thermoelectric figure of merit is still poor. Moreover, most studies so far have focused on the preparation of $p$-type polymers and the optimization of their thermoelectric transport properties via doping and structural engineering. Reliable n-type polymers are still lacking due to their instability in ambient air conditions. To overcome these bottlenecks, hybrids of inorganic/organic semiconductors 
should be a matter of focus as they provide a path to take advantage of the best of both the organic and inorganic worlds.

Despite highlighting exquisite demonstrations of conductive polymers and their hybrids for thermoelectric applications in this review, a better understanding of key influencing factors governing the thermoelectric behavior of organic materials has to be prioritized. For instance, future research on doping mechanisms and interfacial effects in polymeric hybrids can shed light on limitations in current theoretical and experimental thermoelectric systems. In addition, macromolecular engineering of soluble n-type coordination polymers and improved decoupling between the thermal and the electrical conductivities via selectivecharge Anderson localization have to be further explored for transitioning toward efficient organic thermoelectric devices. Distinctly, inherent advantages of sophisticated nanomanufacturing methods to fully control the polymer microstructures in thermopiles should lead to a fine tuning of the electronic band structure, density of states, and thus the thermoelectric performance.

The interest in organic conductive polymers for flexible or even wearable electronics relies on the conservation of their inherent main features when subjected to mechanical or chemical stresses. To our knowledge, there are few studies dealing with such issues. First, regarding the correlation between the mechanical and conductive characteristics, it has been shown that mechanical drawing to fabricate conductive polymer fibers results in an increase of the Young modulus as well as an improvement of the interchain charge transport. This behavior is linked to the enhancement of the chain packing leading to both strain hardening and extended backbone planarity $[237,238]$. However, assessing general relationships between mechanical and electronic characteristics is rather complex, as recently reviewed by Gomez et al. [239]. Yet, bulk mechanical tests or rheology measurements could be efficient tools in order to decipher the interplay between the mechanical behavior and transport properties. In particular, stretchability for wearable devices has to be considered as a strong asset; therefore, a trade-off between the transport properties and the flexibility of the conductive polymer layer is often required. Methods aiming to improve the stretchability of organic conductive polymers include blending with elastomeric materials [240], incorporating flexible moieties or blocks in the macromolecular structure [241, 242], or adding a low fraction of plasticizers [243]. Nevertheless, it should be 
noted that technologies using conjugated polymers often process them as thin films; thus, mechanical testing in a thin film configuration is required to fully characterize their mechanical behavior.

Second, in regards to the chemical stability of $\pi$-conjugated polymers, the field has been recently reviewed by Oh et al. [244], where they identify the susceptibility of conductive polymers to various chemical or physical processes at different stages of their life cycle. They accordingly report some strategies to overcome degradation factors such as oxygen sensitivity, humidity, light or heat. The most common approach to counter-interact the degradation of organic conductive polymers is based on the encapsulation of the active layers by various laminates either preventing the diffusion of detrimental species [245, 246] or acting as UV-filters [247]. Alternatively, molecular and macromolecular design can yield improved stability of $\pi$-conjugated polymers. For instance, tailoring the side groups attached to the $\pi$-conjugated backbone is crucial to prevent $\mathrm{O}_{2}$ or $\mathrm{H}_{2} \mathrm{O}$ susceptibility $[248,249]$. The crosslinking of the polymer layers is also a pathway for the stabilization of organic active layers. In particular, such treatments can result in a higher stability toward solvents, which is a strong asset for the fabrication of multi-layered devices [250, 251].

Finally, development of fully operational TEGs from conductive polymers and their hybrids is necessary. The enhancement of the power output produced is absolutely necessary to assess the "organic and polymeric thermoelectric technology". It is however noteworthy that a direct comparison between the efficiency of the reported devices is not feasible due to their inherent differences in architecture. Therefore, it is crucial to develop standard characterization methods in the thermoelectric community to enable an accurate evaluation of TEGs performance, as is the case in the solar cell community for many years now. Identical device architectures with the same number of legs under a standard thermal gradient have to be used in order to evaluate the open circuit voltage, maximum output power and maximum power density of a TEGs.

Author Contributions: GP and FP prepared the first draft of this work under AP's guidance. AP edited and wrote the final draft. GF, EC, AR, and GH reviewed and provided comments on the final draft. 
Funding: A.P. is thankful for the financial support of Science Foundation Ireland (SFI) under grant no. 18/SIRG/5621.

Acknowledgments: GP, FP, GF, EC and GH acknowledge the support of Université de Bordeaux. AP and AR acknowledge the support of Trinity College Dublin. Authors thank Ms. Juliette Joubert for the graphical abstract design.

Conflicts of Interest: The authors declare no conflicts of interest.

References

1. Breeze, P., Power generation technologies, ed. Elsevier. 2014.

2. Saidi, K. and M.B. Mbarek, Nuclear energy, renewable energy, $\mathrm{CO}_{2}$ emissions, and economic growth for nine developed countries: Evidence from panel Granger causality tests. Prog. Nucl. Energy, 2016. 88: p. 364-374.

3. Smith, G.L., et al., Clouds and earth radiant energy system (CERES), a review: past, present and future. Adv. Space Res., 2011. 48: p. 254.

4. Zhu, J., et al., A review of geothermal energy resources, development, and applications in China: Current status and prospects. Energy, 2015. 93: p. 466.

5. Harde, H., Radiation and heat transfer in the atmosphere: A comprehensive approach on a molecular basis. Int. J. Atmos. Sci., 2013. 2013: p. 26.

6. Laboratory, L.L.N. Energy flow charts. 2019 July 2019]; Available from: https://flowcharts.Ilnl.gov/.

7. Baglione, M.L., Development of system analysis methodologies and tools for modeling and optimizing vehicle system efficiency. 2007, Ph.D. Thesis - University of Michigan.

8. Hanley, S. Electric car myth buster - efficiency 2018 April 2019]; Available from: https://cleantechnica.com/2018/03/10/electric-car-myth-buster-efficiency/.

9. Vining, C.B., An inconvenient truth about thermoelectrics. Nat. Mater., 2009. 8: p. 83.

10. Han, M.-K., et al., Thermoelectric properties of Bi2Te3: Cul and the effect of its doping with $\mathrm{Pb}$ atoms. Materials, 2017. 10(11): p. 1235.

11. Eibl, O., et al., Thermoelectric Bi2Teznanomaterials, ed. Wiley-VCH. 2015.

12. Lowhorn, N.D., et al., Round-robin studies of two potential seebeck coefficient standard reference materials. J. Res. Natl. Inst. Stand. Technol., 2009. 114: p. 37-55.

13. Heeger, A.J., Nobel lecture: Semiconducting and metallic polymers: The fourth generation of polymeric materials. Rev. Mod. Phys., 2001. 73(3): p. 681-700.

14. Heeger, A.J., Semiconducting and metallic polymers: The fourth generation of polymeric materials. J. Phys. Chem. B, 2001. 105(36): p. 8475-8491.

15. Geoghegan, M. and G. Hadziioannou, Polymer electronics ed. O.U. Press. 2019.

16. Russ, B., et al., Organic thermoelectric materials for energy harvesting and temperature control. Nat. Rev. Mater., 2016. 1: p. 16050.

17. Bubnova, O. and X. Crispin, Towards polymer-based organic thermoelectric generators. Energy Environ. Sci., 2012. 5(11): p. 9345-9362. 
18. Wang, X.-S., et al., Investigations on the mechanical properties of conducting polymer coating-substrate structures and their influencing factors. Int. J. Mol. Sci., 2009. 10(12): p. 5257-5284.

19. Le, T.-H., Y. Kim, and H. Yoon, Electrical and electrochemical properties of conducting polymers. Polymers, 2017. 9(4): p. 150.

20. Huang, C., X. Qian, and R. Yang, Thermal conductivity of polymers and polymer nanocomposites. Mater. Sci. Eng., R, 2018. 132: p. 1-22.

21. Magu, T.O., et al., A review on conducting polymers-based composites for energy storage application. Chem. Rev., 2019. 1(1): p. 19-34.

22. Vedernikov, M.V. and E.K. Iordanishvili. A.F. loffe and origin of modern semiconductor thermoelectric energy conversion. in Seventeenth International Conference on Thermoelectrics. ICT98. 1998. IEEE.

23. Lu, N., L. Li, and M. Liuab, A review of carrier thermoelectric-transport theory in organic semiconductors. Phys. Chem. Chem. Phys., 2016. 18: p. 19503-19525.

24. Bates, L., Introduction to solid state physics by C. Kittel. Acta Cryst., 1954. 7(1): p. 144.

25. Lenz, E., Einige versuche im gebiete des galvanismus. Ann. Phys., 1838. 120(6): p. 342-349.

26. Esq, J.P.J., XXXVIII. On the heat evolved by metallic conductors of electricity, and in the cells of a battery during electrolysis. Lond. Edinb. Dubl. Phil. Mag., 1841. 19(124): p. 260-277.

27. Rowe, D.M., Thermoelectrics handbook: Macro to nano, ed. T.a.F. CRC Press Book. 2019. 1022.

28. Reddy Sangi Reddy, P., Transport of charge and energy in metal-molecule-metal junctions. 2007, Ph.D. Thesis - University of California, Berkeley.

29. Wang, R.Y.-S., Energy transport and conversion in nanostructured materials. 2008, Ph.D. Thesis, University of California, Berkeley.

30. Yee, S.K., et al., Thermoelectric power factor optimization in PEDOT:PSS tellurium nanowire hybrid composites. Phys. Chem. Chem. Phys., 2013. 15(11): p. 4024-4032.

31. Snyder, G.J., Thermoelectric energy harvesting, in Energy Harvesting Technologies. 2009, Springer, Boston, MA. p. 325-336.

32. Uher, C., Materials aspect of thermoelectricity. 1 ed. 2016: CRC Press.

33. Fan, Z., et al., Polymer films with ultrahigh thermoelectric properties arising from significant seebeck coefficient enhancement by ion accumulation on surface. Nano Energy, 2018. 51: p. 481-488.

34. Chaikin, P.M., An Introduction to Thermopower for Those Who Might Want to Use It to Study Organic Conductors and Superconductors, in Organic Superconductivity, V.Z. Kresin and W.A. Little, Editors. 1990, Springer US: Boston, MA. p. 101-115.

35. Cutler, M. and N.F. Mott, Observation of Anderson Localization in an Electron Gas. Physical Review, 1969. 181(3): p. 1336-1340.

36. Ashcroft, N.W. and N.D. Mermin, Solid State Physics, Cornell University. 1976, Saunders College Publishing, Harcourt Brace Jovanovich College Publishers ....

37. Guo, X. and A. Facchetti, The journey of conducting polymers from discovery to application. Nature Materials, 2020. 19(9): p. 922-928.

38. Haque, S.M., et al., Electrical properties of different polymeric materials and their applications: The influence of electric field, in Properties and Applications of Polymer Dielectrics, I. Boxue Du, Editor. 2017. 
39. Robbins, A.B. and A.J. Minnich, Crystalline polymers with exceptionally low thermal conductivity studied using molecular dynamics. Appl. Phys. Lett., 2015. 107(20): p. 201908.

40. Choy, C.L., Thermal conductivity of polymers. Polymer, 1977. 18(10): p. 984-1004.

41. Yaws, C.L., Transport properties of chemicals and hydrocarbons, ed. E.s. Edition. 2009: Elsevier. 600.

42. Culebras, M., C.M. Gómez, and A. Cantarero, Review on polymers for thermoelectric applications. Materials, 2014. 7(9): p. 6701-6732.

43. Orrill, M. and S. LeBlanc, Printed thermoelectric materials and devices: Fabrication techniques, advantages, and challenges. J. Appl. Polym. Sci., 2017. 134(3): p. 44256.

44. Chiang, C.K., et al., Electrical conductivity in doped polyacetylene. Phys. Rev. Lett., 1977. 39(17): p. 1098-1101.

45. Su, W.P., J.R. Schrieffer, and A.J. Heeger, Solitons in polyacetylene. Phys. Rev. Lett., 1979. 42(25): p. 1698-1701.

46. Su, W.P. and J.R. Schrieffer, Soliton dynamics in polyacetylene. Proc. Natl. Acad. Sci. U. S. A., 1980. 77(10): p. 5626-5629.

47. Xuan, Y., et al., Thermoelectric properties of conducting polymers: The case of poly(3hexy/thiophene). Phys. Rev. B, 2010. 82(11): p. 115454.

48. Park, Y.W., et al., Semiconductor-metal transition in doped $(\mathrm{CH})_{x}$ : Thermoelectric power. Solid State Commun., 1979. 29(11): p. 747-751.

49. Hiroshige, Y., M. Ookawa, and N. Toshima, Thermoelectric figure-of-merit of iodinedoped copolymer of phenylenevinylene with dialkoxyphenylenevinylene. Synth. Met., 2007. 157(10): p. 467-474.

50. Scholdt, M., et al., Organic semiconductors for thermoelectric applications. J. Electron. Mater., 2010. 39(9): p. 1589-1592.

51. Groenendaal, L., et al., Poly(3,4-ethylenedioxythiophene) and its derivatives: Past, present, and future. Adv. Mater., 2000. 12(7): p. 481-494.

52. Bubnova, O., et al., Optimization of the thermoelectric figure of merit in the conducting polymer poly(3,4-ethylenedioxythiophene). Nat. Mater., 2011. 10(6): p. 429-433.

53. Kirchmeyer, S. and K. Reuter, Scientific importance, properties and growing applications of poly(3,4-ethylenedioxythiophene). J. Mater. Chem., 2005. 15(21): p. 2077-2088.

54. Elschner, A., et al., PEDOT: Principles and applications of an intrinsically conductive polymer, ed. C.P.B.s. edition. 2019. 377.

55. Xia, Y. and J. Ouyang, Significant different conductivities of the two grades of poly $(3,4-$ ethylenedioxythiophene):poly(styrenesulfonate), Clevios $P$ and Clevios PH1000, arising from different molecular weights. ACS Appl. Mater. Interfaces, 2012. 4(8): p. 41314140.

56. Luo, J., et al., Enhancement of the thermoelectric properties of PEDOT:PSS thin films by post-treatment. J. Mater. Chem. A, 2013. 1(26): p. 7576-7583.

57. Xiong, J., et al., Highly electrical and thermoelectric properties of a PEDOT:PSS thinfilm via direct dilution-filtration. RSC Adv., 2015. 5(75): p. 60708-60712.

58. Liu, S., et al., The optimization of thermoelectric properties in a PEDOT:PSS thin film through post-treatment. RSC Adv., 2014. 5(3): p. 1910-1917.

59. Zhang, L., et al., Enhanced thermoelectric properties of PEDOT:PSS films via a novel two-step treatment. RSC Adv., 2015. 5(128): p. 105592-105599. 
60. Zhang, S., et al., Poly(3,4-ethylenedioxythiophene):polystyrene sulfonate films with low conductivity and low acidity through a treatment of their solutions with probe ultrasonication and their application as hole transport layer in polymer solar cells and perovskite solar cells. Org. Electron., 2016. 32: p. 149-156.

61. Xia, Y., et al., Solution-processed highly superparamagnetic and conductive PEDOT:PSS/Fe3O4 nanocomposite films with high transparency and high mechanical flexibility. ACS Appl. Mater. Interfaces, 2017. 9(22): p. 19001-19010.

62. Bae, E.J., et al., Enhancement of thermoelectric properties of PEDOT:PSS and tellurium-PEDOT:PSS hybrid composites by simple chemical treatment. Sci. Rep., 2016. 6: p. 18805.

63. Kong, F., et al., Effect of solution $\mathrm{pH}$ value on thermoelectric performance of freestanding PEDOT:PSS films. Synth. Met., 2013. 185-186: p. 31-37.

64. Yi, C., et al., Enhanced thermoelectric properties of poly $(3,4-$ ethylenedioxythiophene):poly(styrenesulfonate) by binary secondary dopants. ACS Appl. Mater. Interfaces, 2015. 7(17): p. 8984-8989.

65. Mengistie, D.A., et al., Enhanced thermoelectric performance of PEDOT:PSS flexible bulky papers by treatment with secondary dopants. ACS Appl. Mater. Interfaces, 2015. 7(1): p. 94-100.

66. Sun, K., et al., Review on application of PEDOTs and PEDOT:PSS in energy conversion and storage devices. J. Mater. Sci.: Mater. Electron., 2015. 26(7): p. 4438-4462.

67. Xia, Y., et al., Effects of organic inorganic hybrid perovskite materials on the electronic properties and morphology of poly(3,4ethylenedioxythiophene):poly(styrenesulfonate) and the photovoltaic performance of planar perovskite solar cells. J. Mater. Chem. A, 2015. 3(31): p. 15897-15904.

68. Liu, E., et al., Enhanced thermoelectric performance of PEDOT:PSS films by solvent thermal treatment. J. Polym. Res., 2015. 22(12): p. 240.

69. Yu, Z., et al., PEDOT:PSS films with metallic conductivity through a treatment with common organic solutions of organic salts and their application as a transparent electrode of polymer solar cells. ACS Appl. Mater. Interfaces, 2016. 8(18): p. 1162911638.

70. Fan, B., et al., Conducting polymer/carbon nanotube composite as counter electrode of dye-sensitized solar cells. Appl. Phys. Lett., 2008. 93(14): p. 143103.

71. Heywang, G. and F. Jonas, Poly(alkylenedioxythiophene)s-new, very stable conducting polymers. Adv. Mater., 1992. 4: p. 116-118.

72. Zuber, K., et al., Improved PEDOT conductivity via suppression of crystallite formation in Fe(III) tosylate during vapor phase polymerization. Macromol. Rapid Commun., 2008. 29(18): p. 1503-1508.

73. Winther-Jensen, B., D.W. Breiby, and K. West, Base inhibited oxidative polymerization of 3,4-ethylenedioxythiophene with iron(III)tosylate. Synth. Met., 2005. 152(1): p. 1-4.

74. de Leeuw, D.M., et al., Stability of n-type doped conducting polymers and consequences for polymeric microelectronic devices. Synth. Met., 1997. 87(1): p. 5359.

75. Fabretto, M., et al., High conductivity PEDOT resulting from glycol/oxidant complex and glycol/polymer intercalation during vacuum vapour phase polymerisation. Polymer, 2011. 52: p. 1725-1730.

76. Noriega, R., et al., A general relationship between disorder, aggregation and charge transport in conjugated polymers. Nat. Mater., 2013. 12(11): p. 1038-1044. 
77. Petsagkourakis, I., et al., Correlating the Seebeck coefficient of thermoelectric polymer thin films to their charge transport mechanism. Org. Electron., 2018. 52: p. 335-341.

78. Kaiser, A.B. and V. Skákalová, Electronic conduction in polymers, carbon nanotubes and graphene. Chem. Soc. Rev., 2011. 40(7): p. 3786-3801.

79. Kim, J.Y., et al., Enhancement of electrical conductivity of poly $(3,4-$ ethylenedioxythiophene)/poly(4-styrenesulfonate) by a change of solvents. Synth. Met., 2002. 126(2-3): p. 311-316.

80. Li, J., et al., Synthesis and thermoelectric properties of hydrochloric acid-doped polyaniline. Synth. Met., 2010. 160(11): p. 1153-1158.

81. Kong, F.-F., et al., Simultaneous enhancement of electrical conductivity and Seebeck coefficient of poly(3,4-ethylenedioxythiophene):poly(styrenesulfonate) films treated with urea. Chin. Phys. Lett., 2011. 28(3): p. 037201.

82. Elschner, A., et al., PEDOT: principles and applications of an intrinsically conductive polymer. 2010: CRC press.

83. Kim, G.H., et al., Engineered doping of organic semiconductors for enhanced thermoelectric efficiency. Nat. Mater., 2013. 12(8): p. 719-723.

84. Palumbiny, C.M., et al., The crystallization of PEDOT:PSS polymeric electrodes probed in situ during printing. Adv. Mater., 2015. 27: p. 3391-3397.

85. Bubnova, O., et al., Semi-metallic polymers. Nat. Mater., 2014. 13(2): p. 190-194.

86. Kishi, N., et al., Enhancement of thermoelectric properties of PEDOT:PSS thin films by addition of anionic surfactants. J. Mater. Sci.: Mater. Electron., 2018. 29(5): p. 40304034.

87. Wang, J., K. Cai, and S. Shen, Enhanced thermoelectric properties of poly $(3,4-$ ethylenedioxythiophene) thin films treated with $\mathrm{H}_{2} \mathrm{SO}_{4}$ Org. Electron., 2014. 15(11): p. 3087-3095.

88. Beretta, D., et al., Thermoelectric properties of highly conductive poly(3,4ethylenedioxythiophene) polystyrene sulfonate printed thin films. ACS Appl. Mater. Interfaces, 2017. 9(21): p. 18151-18160.

89. Han, Y., Enhanced electrical properties of PEDOT:PSS via synergistic effect. Soft Mater., 2018. 16(1): p. 31-36.

90. Lee, S.H., et al., Novel solution-processable, dedoped semiconductors for application in thermoelectric devices. J. Mater. Chem. A, 2014. 2(33): p. 13380-13387.

91. Massonnet, N., et al., Improvement of the Seebeck coefficient of PEDOT:PSS by chemical reduction combined with a novel method for its transfer using free-standing thin films. J. Mater. Chem. C, 2014. 2(7): p. 1278-1283.

92. Khan, Z.U., et al., Acido-basic control of the thermoelectric properties of poly(3,4ethylenedioxythiophene)tosylate (PEDOT-Tos) thin films. J. Mater. Chem. C, 2015. 3(40): p. 10616-10623.

93. Fan, Z., et al., Significantly enhanced thermoelectric properties of PEDOT:PSS films through sequential post-treatments with common acids and bases. Adv. Energy Mater., 2017. 7(8): p. 1602116.

94. Gurunathan, K., et al., Electrochemically synthesised conducting polymeric materials for applications towards technology in electronics, optoelectronics and energy storage devices. Mater. Chem. Phys., 1999. 61(3): p. 173-191.

95. Zhang, L., et al., Thermoelectric properties of PEDOT films prepared by electrochemical polymerization. J. Polym. Sci. Pol. Phys., 2017. 55(6): p. 524-531. 
96. Park, T., et al., Flexible PEDOT electrodes with large thermoelectric power factors to generate electricity by the touch of fingertips. Energy Environ. Sci., 2013. 6(3): p. 788792.

97. Bubnova, O., M. Berggren, and X. Crispin, Tuning the thermoelectric properties of conducting polymers in an electrochemical transistor. J. Am. Chem. Soc., 2012. 134(40): p. 16456-16459.

98. Brédas, J.L., et al., Highly conducting polyparaphenylene, polypyrrole, and polythiophene chains: An ab initio study of the geometry and electronic-structure modifications upon doping. Phys. Rev. B, 1984. 29(12): p. 6761-6773.

99. Chance, R.R., J.L. Brédas, and R. Silbey, Bipolaron transport in doped conjugated polymers. Phys. Rev. B, 1984. 29(8): p. 4491-4495.

100. Basescu, N., et al., High electrical conductivity in doped polyacetylene. Nature, 1987. 327(6121): p. 403-405.

101. Zuzok, R., et al., Thermoelectric power and conductivity of iodine-doped "new" polyacetylene. J. Chem. Phys., 1991. 95(2): p. 1270-1275.

102. Park, Y.W., et al., Metallic properties of transition metal halides doped polyacetylene: the soliton liquid state. Synth. Met., 1991. 41(1): p. 27-32.

103. Kaneko, H., et al., Magnetoresistance and thermoelectric power studies of metalnonmetal transition in iodine-doped polyacetylene. Synth. Met., 1993. 57(2): p. 49004905.

104. Park, Y.W., et al., Thermopower and conductivity of metallic polyaniline. Solid State Commun., 1987. 63: p. 1063-1066.

105. Park, Y.W., et al., Electrical properties of polyaniline and substituted polyaniline derivatives. Synth. Met., 1989. 29(1): p. 389-394.

106. Dalas, E., S. Sakkopoulos, and E. Vitoratos, Chemical preparation, direct-current conductivity and thermopower of polyaniline and polypyrrole composites. J. Mater. Sci., 1994. 29(15): p. 4131-4133.

107. Bhadra, S., N.K. Singha, and D. Khastgir, Electrochemical synthesis of polyaniline and its comparison with chemically synthesized polyaniline. J. Appl. Polym. Sci., 2007. 104(3): p. 1900-1904.

108. Yan, H., N. Sada, and N. Toshima, Thermal transporting properties of electrically conductive polyaniline films as organic thermoelectric materials. J. Therm. Anal. Calorim., 2002. 69(3): p. 881-887.

109. Abad, B., et al., Improved power factor of polyaniline nanocomposites with exfoliated graphene nanoplatelets (GNPS). J. Mater. Chem. A, 2013. 1(35): p. 10450-10457.

110. Nath, C., et al., High thermoelectric figure of merit in nanocrystalline polyaniline at low temperatures. Appl. Phys. Lett., 2014. 105(13): p. 133108.

111. Yang, Y., S. Chen, and L. Xu, Enhanced conductivity of polyaniline by conjugated crosslinking. Macromol. Rapid Commun., 2011. 32(7): p. 593-597.

112. Armes, S.P., Optimum reaction conditions for the polymerization of pyrrole by iron(III) chloride in aqueous solution. Synth. Met., 1987. 20(3): p. 365-371.

113. Qi, G., L. Huang, and H. Wang, Highly conductive free standing polypyrrole films prepared by freezing interfacial polymerization. ChemComm., 2012. 48(66): p. 82468248.

114. Jha, P., et al., Growth of free-standing polypyrrole nanosheets at air/liquid interface using J-aggregate of porphyrin derivative as in-titu template. Macromolecules, 2011. 44(12): p. 4583-4585. 
115. Oukil, D., et al., Electrochemical synthesis of polypyrrole films doped by ferrocyanide ions onto iron substrate: Application in the electroanalytical determination of uric acid. Sens. Actuator B-Chem., 2014. 204: p. 203-210.

116. Kemp, N.T., et al., Thermoelectric power and conductivity of different types of polypyrrole. J. Polym. Sci. Pol. Phys., 1999. 37(9): p. 953-960.

117. Culebras, M., et al., Controlling the thermoelectric properties of polymers: application to PEDOT and polypyrrole. Phys. Chem. Chem. Phys., 2015. 17(23): p. 15140-15145.

118. Endrődi, B., et al., Molecular and supramolecular parameters dictating the thermoelectric performance of conducting polymers: A case study using poly(3alkylthiophene)s. J. Phys. Chem. C, 2015. 119(16): p. 8472-8479.

119. Zhang, Q., et al., Thermoelectric energy from flexible P3HT films doped with a ferric salt of triflimide anions. Energy Environ. Sci., 2012. 5(11): p. 9639-9644.

120. Duong, D.T., et al., Direct observation of doping sites in temperature-controlled, $p$ doped P3HT thin films by conducting atomic force microscopy. Adv. Mater., 2014. 26(35): p. 6069-6073.

121. Jacobs, I.E., et al., Comparison of solution-mixed and sequentially processed P3HT:F4TCNQ films: Effect of doping-induced aggregation on film morphology. J. Mater. Chem. C, 2016. 4(16): p. 3454-3466.

122. Wang, C., et al., Optical measurement of doping efficiency in poly(3-hexylthiophene) solutions and thin films. Phys. Rev. B, 2015. 91(8): p. 085205.

123. Hynynen, J., et al., Enhanced electrical conductivity of molecularly p-doped poly(3hexylthiophene) through understanding the correlation with solid-state order. Macromolecules, 2017. 50(20): p. 8140-8148.

124. Bolton, J. and J. Rzayev, Synthesis and melt self-assembly of PS-PMMA-PLA triblock bottlebrush copolymers. Macromolecules, 2014. 47(9): p. 2864-2874.

125. Cochran, J.E., et al., Molecular interactions and ordering in electrically doped polymers: blends of PBTTT and F4TCNQ. Macromolecules, 2014. 47(19): p. 6836-6846.

126. Sun, J., et al., Simultaneous increase in Seebeck coefficient and conductivity in a doped poly(alkylthiophene) blend with defined density of states. Macromolecules, 2010. 43(6): p. 2897-2903.

127. Zuo, G., et al., Molecular doping and trap filling in organic semiconductor host-guest systems. J. Phys. Chem. C, 2017. 121(14): p. 7767-7775.

128. Scholes, D.T., et al., Overcoming film quality issues for conjugated polymers doped with F4TCNQ by solution sequential processing: Hall effect, structural, and optical measurements. J. Phys. Chem. Lett., 2015. 6(23): p. 4786-4793.

129. Zuo, G., et al., High thermoelectric power factor from multilayer solution-processed organic films. Appl. Phys. Lett., 2018. 112(8): p. 083303.

130. Zhang, Q., et al., Effects of structural order in the pristine state on the thermoelectric power-factor of doped PBTTT films. Synth. Met., 2012. 162(9): p. 788-793.

131. Zhang, Q., et al., What to expect from conducting polymers on the playground of thermoelectricity: Lessons learned from four high-mobility polymeric semiconductors. Macromolecules, 2014. 47(2): p. 609-615.

132. Glaudell, A.M., et al., Impact of the doping method on conductivity and thermopower in semiconducting polythiophenes. Adv. Energy Mater., 2015. 5(4): p. 1401072.

133. Patel, S.N., et al., Morphology controls the thermoelectric power factor of a doped semiconducting polymer. Sci. Adv., 2017. 3(6): p. e1700434. 
134. Patel, S.N., et al., Increasing the thermoelectric power factor of a semiconducting polymer by doping from the vapor phase. ACS Macro Lett., 2016. 5(3): p. 268-272.

135. Lim, E., et al., Thermoelectric properties of poly(3-hexylthiophene) (P3HT) doped with 2,3,5,6-tetrafluoro-7,7,8,8-tetracyanoquinodimethane (F4TCNQ) by vapor-phase infiltration. Chem. Mater., 2018. 30(3): p. 998-1010.

136. Aïch, R.B., et al., Electrical and thermoelectric properties of poly(2,7-Carbazole) derivatives. Chem. Mater., 2009. 21(4): p. 751-757.

137. Wang, L., et al., The effect of the backbone structure on the thermoelectric properties of donor-acceptor conjugated polymers. Polym. Chem., 2017. 8(32): p. 4644-4650.

138. Jung, Y.-J., et al., Heat-killed Lactobacillus casei confers broad protection against influenza $A$ virus primary infection and develops heterosubtypic immunity against future secondary infection. Sci. Rep., 2017. 7(1): p. 17360.

139. Lüssem, B., et al., Doped organic transistors. Chem. Rev., 2016. 116(22): p. 1371413751.

140. Naab, B.D., et al., Mechanistic Study on the Solution-Phase n-Doping of 1, 3-Dimethyl2-aryl-2, 3-dihydro-1 H-benzoimidazole Derivatives. Journal of the American Chemical Society, 2013. 135(40): p. 15018-15025.

141. Li, C.Z., et al., Doping of fullerenes via anion-induced electron transfer and its implication for surfactant facilitated high performance polymer solar cells. Advanced materials, 2013. 25(32): p. 4425-4430.

142. Nollau, A., et al., Controlled n-type doping of a molecular organic semiconductor: Naphthalenetetracarboxylic dianhydride (NTCDA) doped with bis(ethylenedithio)tetrathiafulvalene (BEDT-TTF). J. Appl. Phys., 2000. 87(9): p. 4340-4343.

143. Wong, W.W.H., et al., Benzotriazole-based donor-acceptor conjugated polymers with a broad absorption in the visible range. Polym. Chem., 2014. 5(4): p. 1258-1263.

144. Schmidt, R., et al., High-performance air-stable n-channel organic thin film transistors based on halogenated perylene bisimide semiconductors. J. Am. Chem. Soc., 2009. 131(17): p. 6215-6228.

145. Fukutomi, Y., et al., Naphthodithiophenediimide (NDTI): Synthesis, structure, and applications. J. Am. Chem. Soc., 2013. 135(31): p. 11445-11448.

146. Menke, T., et al., In-situ conductivity and Seebeck measurements of highly efficient ndopants in fullerene C60 Appl. Phys. Lett., 2012. 100(9): p. 093304.

147. Wei, P., et al., Use of a $1 \mathrm{H}$-benzoimidazole derivative as an $n$-type dopant and to enable air-stable solution-processed $n$-channel organic thin-film transistors. J. Am. Chem. Soc., 2010. 132(26): p. 8852-8853.

148. Weber, C.D., C. Bradley, and M.C. Lonergan, Solution phase $n$-doping of C6oand PCBM using tetrabutylammonium fluoride. J. Mater. Chem. A, 2013. 2(2): p. 303-307.

149. Lu, J., et al., Amphoteric and controllable doping of carbon nanotubes by encapsulation of organic and organometallic molecules. Phys. Rev. Lett., 2004. 93(11): p. 116804.

150. Liu, J., et al., Doping engineering enables highly conductive and thermally stable $n$ type organic thermoelectrics with high power factor. ACS Appl. Energy Mater., 2019. 2(9): p. 6664-6671.

151. Moses, D., et al., Inter-soliton electron hopping transport in trans- $(\mathrm{CH})_{X}$. Solid State Commun., 1981. 40(11): p. 1007-1010.

152. Reynolds, J.R., J.C.W. Chien, and C.P. Lillya, Intrinsically electrically conducting poly(metal tetrathiooxalates). Macromolecules, 1987. 20: p. 1184. 
153. Reynolds, J.R., et al., Electrically conducting transition metal complexes of tetrathiooxalate. J. Chem. Soc. Chem. Commun. , 1985: p. 268.

154. Faulmann, C., et al., Nickel ethylene tetrathiolate polymers as nanoparticles: A new synthesis for future applications? J. Nanoparticle Res., 2013. 15(4): p. 1-18.

155. Oshima, K., et al., Hybrid-type organic thermoelectric materials containing nanoparticles as a carrier transport promoter. J. Electron. Mater., 2017. 46(5): p. 3207-3214.

156. Sun, Y., et al., Optimization of the thermoelectric properties of poly(nickelethylenetetrathiolate) synthesized via potentiostatic deposition. Sci. China Chem., 2016. 59(10): p. 1323-1329.

157. Sun, Y., et al., Organic Thermoelectric Materials and Devices Based on p-and n-Type Poly (metal 1, 1, 2, 2-ethenetetrathiolate) s. Advanced Materials, 2012. 24(7): p. 932937.

158. Sun, Y., et al., Flexible n-type high-performance thermoelectric thin films of poly(nickel-ethylenetetrathiolate) prepared by an electrochemical method. Adv. Mater., 2016. 28: p. 3351-3358.

159. Huang, X., et al., A two-dimensional $\pi$ - $d$ conjugated coordination polymer with extremely high electrical conductivity and ambipolar transport behaviour. Nat. Commun., 2015. 6: p. 7408.

160. Lei, T., et al., Electron-deficient poly(p-phenylene vinylene) provides electron mobility over $1 \mathrm{~cm}^{2} V^{-1} \mathrm{~s}^{-1}$ under ambient conditions. J. Am. Chem. Soc., 2013. 135(33): p. 12168-12171.

161. Shi, K., et al., Toward high performance n-type thermoelectric materials by rational modification of BDPPV backbones. J. Am. Chem. Soc., 2015. 137(22): p. 6979-6982.

162. Ma, W., et al., Enhanced molecular packing of a conjugated polymer with high organic thermoelectric power factor. ACS Appl. Mater. Interfaces, 2016. 8(37): p. 24737-24743.

163. Zhao, X., et al., High conductivity and electron-transfer validation in an n-type fluoride-anion-doped polymer for thermoelectrics in air. Adv. Mater., 2017. 29(34): p. 1606928.

164. Lu, Y., et al., Persistent Conjugated Backbone and Disordered Lamellar Packing Impart Polymers with Efficient $n$-Doping and High Conductivities. Advanced Materials, 2021. 33(2): p. 2005946.

165. Schlitz, R.A., et al., Solubility-limited extrinsic n-type doping of a high electron mobility polymer for thermoelectric applications. Adv. Mater., 2014. 26(18): p. 2825-2830.

166. Liu, J., et al., Enhancing molecular n-type doping of donor-acceptor copolymers by tailoring side chains. Adv. Mater., 2018. 30(7): p. 1704630.

167. Kiefer, D., et al., Enhanced n-doping efficiency of a naphthalenediimide-based copolymer through polar side chains for organic thermoelectrics. ACS Energy Lett., 2018. 3(2): p. 278-285.

168. Wang, Y., et al., Naphthodithiophenediimide-benzobisthiadiazole-based polymers: versatile n-type materials for field-effect transistors and thermoelectric devices. Macromolecules, 2017. 50(3): p. 857-864.

169. Liu, J., et al., Overcoming Coulomb interaction improves free-charge generation and thermoelectric properties for $n$-doped conjugated polymers. ACS Energy Letters, 2019. 4(7): p. 1556-1564. 
170. Wang, S., et al., Thermoelectric properties of solution-processed n-doped ladder-type conducting polymers. Adv. Mater., 2016. 28(48): p. 10764-10771.

171. Abdalla, H., G. Zuo, and M. Kemerink, Range and energetics of charge hopping in organic semiconductors. Physical Review B, 2017. 96(24): p. 241202.

172. Lepinoy, M., et al., Thermopower scaling in conducting polymers. Scientific Reports, 2020. 10(1): p. 8086.

173. Beretta, D., et al., Thermoelectrics: From history, a window to the future. Mater. Sci. Eng., R, 2019. 138: p. 100501.

174. Oshima, K., et al., Thermostability of hybrid thermoelectric materials consisting of poly(Ni-ethenetetrathiolate), polyimide and carbon nanotubes. Materials, 2017. 10(7): p. 824.

175. Zhang, K., J. Qiu, and S. Wang, Thermoelectric properties of PEDOT nanowire/PEDOT hybrids. Nanoscale, 2016. 8(15): p. 8033-8041.

176. Zuo, G., et al., High Seebeck coefficient in mixtures of conjugated polymers. Adv. Funct. Mater., 2018. 28(15): p. 1703280.

177. Abramson, A.R., et al., Fabrication and characterization of a nanowire/polymer-based nanocomposite for a prototype thermoelectric device J. Microelectromech. Syst. , 2004. 13: p. 505-513.

178. He, M., et al., Thermopower enhancement in conducting polymer nanocomposites via carrier energy scattering at the organic-inorganic semiconductor interface. Energy Environ. Sci., 2012. 5(8): p. 8351-8358.

179. Choi, J., et al., High-performance thermoelectric paper based on double carrierfiltering processes at nanowire heterojunctions. Adv. Energy Mater., 2016. 6(9): p. 1502181.

180. Liang, Z., et al., Increased power factors of organic-inorganic nanocomposite thermoelectric materials and the role of energy filtering. J. Mater. Chem. A, 2017. 5(30): p. 15891-15900.

181. Zhang, B., et al., Promising thermoelectric properties of commercial PEDOT:PSS materials and their $\mathrm{Bi}_{2} \mathrm{Te}_{3}$ powder composites. ACS Appl. Mater. Interfaces, 2010. 2(11): p. 3170-3178.

182. McGrail, B.T., A. Sehirlioglu, and E. Pentzer, Polymer composites for thermoelectric applications. Angew. Chem., 2015. 54(6): p. 1710-1723.

183. Du, Y., et al., Simultaneous increase in conductivity and Seebeck coefficient in a polyaniline/graphene nanosheets thermoelectric nanocomposite. Synth. Met., 2012. 161(23): p. 2688-2692.

184. Coates, N.E., et al., Effect of interfacial properties on polymer-nanocrystal thermoelectric transport. Adv. Mater., 2013. 25: p. 1629-1633.

185. Ju, H. and J. Kim, Fabrication of conductive polymer/inorganic nanoparticles composite films: PEDOT:PSS with exfoliated tin selenide nanosheets for polymerbased thermoelectric devices. Chem. Eng. J., 2016. 297: p. 66-73.

186. Wang, L., et al., Exceptional thermoelectric properties of flexible organic- inorganic hybrids with monodispersed and periodic nanophase. Nature communications, 2018. 9(1): p. 1-8.

187. Hu, S., et al., Flexible and high performance of $n$-type thermoelectric PVDF composite film induced by nickel nanowires. Materials \& Design, 2020. 188: p. 108496.

188. Tasis, D., et al., Chemistry of carbon nanotubes. Chem. Rev., 2006. 106(3): p. 11051136. 
189. Soldano, C., A. Mahmood, and E. Dujardin, Production, properties and potential of graphene. Carbon, 2010. 48(8): p. 2127-2150.

190. MacLeod, B.A., et al., Large $n$ - and p-type thermoelectric power factors from doped semiconducting single-walled carbon nanotube thin films. Energy Environ. Sci., 2017. 10(10): p. 2168-2179.

191. Zhang, K., Y. Zhang, and S. Wang, Enhancing thermoelectric properties of organic composites through hierarchical nanostructures. Sci. Rep., 2013. 3: p. 3448.

192. Cho, C., et al., Completely organic multilayer thin film with thermoelectric power factor rivaling inorganic tellurides. Adv. Mater., 2015. 27(19): p. 2996-3001.

193. Cho, C., et al., Stable n-type thermoelectric multilayer thin films with high power factor from carbonaceous nanofillers. Nano Energy, 2016. 28: p. 426-432.

194. Kim, D., et al., Improved thermoelectric behavior of nanotube-filled polymer composites with poly(3,4-ethylenedioxythiophene) poly(styrenesulfonate). ACS nano, 2010. 4(1): p. 513-523.

195. Yu, C., et al., Thermoelectric behavior of segregated-network polymer nanocomposites. Nano Letters, 2008. 8(12): p. 4428-4432.

196. Moriarty, G.P., et al., Thermoelectric behavior of organic thin film nanocomposites. J. Polym. Sci. Pol. Phys., 2012. 51: p. 119-123.

197. Hsu, J.-H., et al., Origin of unusual thermoelectric transport behaviors in carbon nanotube filled polymer composites after solvent/acid treatments. Org. Electron., 2017. 45: p. 182-189.

198. Xu, K., G. Chen, and D. Qiu, Convenient construction of poly $(3,4-$ ethylenedioxythiophene)-graphene pie-like structure with enhanced thermoelectric performance. J. Mater. Chem. A, 2013. 1(40): p. 12395-12399.

199. Hu, X., G. Chen, and X. Wang, An unusual coral-like morphology for composites of poly(3,4-ethylenedioxythiophene)/carbon nanotube and the enhanced thermoelectric performance. Compos. Sci. Technol., 2017. 144: p. 43-50.

200. Yao, Q., et al., Enhanced thermoelectric performance of single-walled carbon nanotubes/polyaniline hybrid nanocomposites. ACS nano, 2010. 4(4): p. 2445-2451.

201. Liang, L., et al., Large-area, stretchable, super flexible and mechanically stable thermoelectric films of polymer/carbon nanotube composites. J. Mater. Chem. C, 2016. 4(3): p. 526-532.

202. Meng, C., C. Liu, and S. Fan, A promising approach to enhanced thermoelectric properties using carbon nanotube networks. Adv. Mater., 2010. 22(4): p. 535-539.

203. Sun, Y., et al., Flexible n-Type High-Performance Thermoelectric Thin Films of Poly (nickel-ethylenetetrathiolate) Prepared by an Electrochemical Method. Advanced materials, 2016. 28(17): p. 3351-3358.

204. Fang, H., et al., Large-scale integration of flexible materials into rolled and corrugated thermoelectric modules. J. Appl. Polym. Sci., 2017. 134(3): p. 44208.

205. Hwang, S., et al., Processing and doping of thick polymer active layers for flexible organic thermoelectric modules. Org. Electron., 2016. 31: p. 31-40.

206. Uchida, K., et al., Longitudinal spin Seebeck effect: From fundamentals to applications. J. Phys. Condens. Matter., 2014. 26(34): p. 343202.

207. Jovovic, V., Thermoelectric waste heat recovery program for passenger vehicles, in Environ. Sci. 2015, Gentherm Incorporated, Azusa, CA (United States).

208. Gordiz, K., A.K. Menon, and S.K. Yee, Interconnect patterns for printed organic thermoelectric devices with large fill factors. J. Appl. Phys., 2017. 122(12): p. 124507. 
209. Menon, A.K., et al., Radial thermoelectric generator fabricated from $n$ - and $p$-type conducting polymers. J. Appl. Polym. Sci., 2017. 134(3).

210. Menon, A.K. and S.K. Yee, Design of a polymer thermoelectric generator using radial architecture. J. Appl. Phys., 2016. 119(5): p. 055501.

211. Jiao, F., et al., Inkjet-printed flexible organic thin-film thermoelectric devices based on p-and n-type poly(metal 1,1,2,2-ethenetetrathiolate)s/polymer composites through ball-milling. Phil. Trans. R. Soc. A., 2014. 372: p. 20130008.

212. Yun, J.H., S.-J. Sung, and K.Y. Cho, Simultaneous fabrication of an alignment layer and a wall structure for a liquid crystal display by solvent-assisted micromolding. Macromol. Res., 2012. 20(5): p. 453-458.

213. ReportBuyer. Thermoelectric energy harvesting 2016-2026: Technologies, devices \& applications for thermoelectric generators. 2016 [cited 2016 July 2019]; Available from: https://www.prnewswire.com/news-releases/thermoelectric-energyharvesting-2016-2026--technologies-devices--applications-for-thermoelectricgenerators-300340880.html.

214. Meulen, R.v.d. Gartner says 8.4 Billion sonnected "things" will be in use in 2017, up 31 percent from 2016. 2017 [cited 2017 July 2019]; Available from: https://www.gartner.com/en/newsroom/press-releases/2017-02-07-gartner-says-8billion-connected-things-will-be-in-use-in-2017-up-31-percent-from-2016.

215. Kishita, Y., et al., Evaluating the life cycle $\mathrm{CO}_{2}$ emissions and costs of thermoelectric generators for passenger automobiles: a scenario analysis. J. Clean. Prod., 2016. 126: p. 607-619.

216. Wei, Q., et al., Polymer thermoelectric modules screen-printed on paper. RSC Adv., 2014. 4(54): p. 28802-28806.

217. Aranguren, P., et al., Optimized design for flexible polymer thermoelectric generators. Appl. Therm. Eng., 2016. 102: p. 402-411.

218. Lee, W., et al., Acidity-controlled conducting polymer films for organic thermoelectric devices with horizontal and vertical architectures. Sci. Rep., 2016. 6(1): p. 33795.

219. Kim, D., D. Ju, and K. Cho, Heat-sink-free flexible organic thermoelectric generator vertically operating with chevron structure. Adv. Mater. Technol., 2018. 3(4): p. 1700335.

220. Du, Y., et al., Thermoelectric fabrics: Toward power generating clothing. Sci. Rep., 2015. 5: p. 6411.

221. Ryan, J.D., et al., Machine-washable PEDOT:PSS dyed silk yarns for electronic textiles. ACS Appl. Mater. Interfaces, 2017. 9(10): p. 9045-9050.

222. Li, Z., et al., A free-standing high-output power density thermoelectric device based on structure-ordered PEDOT:PSS. Adv. Electron. Mater., 2018: p. 1700496.

223. Satoh, N., et al., Organic $\pi$-type thermoelectric module supported by photolithographic mold: A working hypothesis of sticky thermoelectric materials. Sci. Technol. Adv. Mater., 2018. 19(1): p. 517-525.

224. Dong, X., et al., Flexible and transparent organic-inorganic hybrid thermoelectric modules. ACS Appl. Mater. Interfaces, 2018. 10(31): p. 26687-26693.

225. Ferhat, S., et al., Flexible thermoelectric device based on TiSz $(H A)_{x}$ n-type nanocomposite printed on paper. Org. Electron., 2019. 68: p. 256-263.

226. Tian, R., et al., A solution-processed TiS2/organic hybrid superlattice film towards flexible thermoelectric devices. J. Mater. Chem. A, 2017. 5(2): p. 564-570. 
227. Jia, Y., et al., An efficient PEDOT-coated textile for wearable thermoelectric generators and strain sensors. J. Mater. Chem. C, 2019. 7(12): p. 3496-3502.

228. Allison, L.K. and T.L. Andrew, A wearable all-fabric thermoelectric generator. Adv. Mater. Technol., 2019. 4(5): p. 1800615.

229. Leonov, V., Thermoelectric energy harvesting of human body heat for wearable sensors. IEEE Sensors Journal, 2013. 13(6): p. 2284-2291.

230. Qi, Y. and M.C. McAlpine, Nanotechnology-enabled flexible and biocompatible energy harvesting. Energy Environ. Sci., 2010. 3(9): p. 1275-1285.

231. Leonov, V. and R. Vullers, Wearable thermoelectric generators for body-powered devices. J. Electron. Mater., 2008. 38: p. 1491.

232. Mostafalu, P., et al., A toolkit of thread-based microfluidics, sensors, and electronics for 3D tissue embedding for medical diagnostics. Microsyst. Nanoeng., 2016. 2(1): p. 16039.

233. Jia, Y., et al., Effects of additives and post-treatment on the thermoelectric performance of vapor-phase polymerized PEDOT films. J. Polym. Sci. Pol. Phys., 2017. 55(23): p. 1738-1744.

234. Brooke, R., et al., Recent advances in the synthesis of conducting polymers from the vapour phase. Prog. Mater. Sci., 2017. 86: p. 127-146.

235. Hu, E., A. Kaynak, and Y. Li, Development of a cooling fabric from conducting polymer coated fibres: Proof of concept. Synth. Met., 2005. 150: p. 139-143.

236. Jin, W., et al., Exploring Peltier effect in organic thermoelectric films. Nat. Comm., 2018. 9: p. 3586.

237. Cao, Y., P. Smith, and A.J. Heeger, Mechanical and electrical properties of polyacetylene films oriented by tensile drawing. Polymer, 1991. 32(7): p. 1210-1218.

238. Machado, J., M. Masse, and F. Karasz, Anisotropic mechanical properties of uniaxially oriented electrically conducting poly (p-phenylene vinylene). Polymer, 1989. 30(11): p. 1992-1996.

239. Xie, R., R.H. Colby, and E.D. Gomez, Connecting the mechanical and conductive properties of conjugated polymers. Advanced Electronic Materials, 2018. 4(10): p. 1700356.

240. Goffri, S., et al., Multicomponent semiconducting polymer systems with low crystallization-induced percolation threshold. Nature materials, 2006. 5(12): p. 950956.

241. Müller, C., et al., Tough, semiconducting polyethylene-poly (3-hexylthiophene) diblock copolymers. Advanced Functional Materials, 2007. 17(15): p. 2674-2679.

242. Kim, H.J., et al., Architectural engineering of rod-coil compatibilizers for producing mechanically and thermally stable polymer solar cells. ACS nano, 2014. 8(10): p. 10461-10470.

243. Savagatrup, S., et al., Mechanical properties of conjugated polymers and polymerfullerene composites as a function of molecular structure. Advanced Functional Materials, 2014. 24(8): p. 1169-1181.

244. Lee, E.K., et al., Toward environmentally robust organic electronics: approaches and applications. Advanced Materials, 2017. 29(44): p. 1703638.

245. Garcia-Belmonte, G., et al., Oxygen doping-induced photogeneration loss in P3HT: PCBM solar cells. 2012.

246. $\mathrm{Yu}, \mathrm{D}$., et al., Recent progress on thin-film encapsulation technologies for organic electronic devices. Optics Communications, 2016. 362: p. 43-49. 
247. Djurišić, A., et al., Stability issues of the next generation solar cells. physica status solidi (RRL)-Rapid Research Letters, 2016. 10(4): p. 281-299.

248. Han, A.-R., et al., Siloxane side chains: a universal tool for practical applications of organic field-effect transistors. Macromolecules, 2016. 49(10): p. 3739-3748.

249. Roberts, M.E., et al., Water-stable organic transistors and their application in chemical and biological sensors. Proceedings of the National Academy of Sciences, 2008. 105(34): p. 12134-12139.

250. Lee, M.Y., et al., Highly Sensitive and Selective Liquid-Phase Sensors Based on a Solvent-Resistant Organic-Transistor Platform. Advanced Materials, 2015. 27(9): p. 1540-1546.

251. Kim, H.J., et al., Solvent-resistant organic transistors and thermally stable organic photovoltaics based on cross-linkable conjugated polymers. Chemistry of materials, 2012. 24(1): p. 215-221. 